\title{
Löß-Paläoboden-Sequenzen Zentralasiens als Indikatoren einer globalen Klimageschichte des Quartärs?
}

\author{
ARNT BRONGER*) \\ - Loess, Glacial-interglacial cycles, pedostratigraphical correlation, paleosol, Central Asia -
}

\begin{abstract}
Kurzfassung: Eine sehr ähnliche Geländeposition der Moränen um $1850 \mathrm{zu}$ den heutigen Gletschern z. B. in den Alpen, in den südöstlichen kanadischen Rockies oder im Tien-shan als Ergebnis einer geringen Abnahme der Jahresmitteltemperatur führt zu der Hypothese, daß wesentlich größere quartäre Klimaschwankungen im Bereich von 105 Jahren (Glazial-Interglazial-Zyklus) und sogar von $10^{4}$ Jahren (etwa der Länge eines Interglazials) weitestgehend gleichzeitig, jedenfalls im gemäßigten Klimabereich der Nordhemisphäre stattgefunden haben. Dieses Konzept ist wichtig für kontinentale pedostratigraphische Korrelationen: wenn eine Löß-Paläoboden-Abfolge der Brunhes-Epoche mit der 2180-Tiefseekurve des gleichen Zeitabschnitts in gute Übereinstimmung gebracht werden kann, so müßte man Löß-Paläoboden-Sequenzen des Mittel- und Jungpleistozäns selbst in verschiedenen Kontinenten miteinander korrelieren, insbesondere pedostratigraphische Lücken feststellen können. Das würde dann die Rekonstruktion einer Klimageschichte der letzten 800.000 Jahre für den gemäßigten Klimabereich der Nordhemisphäre erlauben.
\end{abstract}

Für eine Löß-Boden-Stratigraphie und insbesondere für paläoklimatische Rückschlüsse ist es einerseits notwendig, die Genese der Paläoböden über seine Merkmale zu erschließen. Die meisten Paläoböden sind aber gekappt und vom hangenden Löß sekundär aufgekalkt. Die Bodenmikromorphologie erlaubt jedoch zwischen primären und sekundären Carbonaten zu unterscheiden und z. B. den Prozeß der Tonverlagerung zweifelsfrei zu erkennen. Dies erlaubt die Trennung von typischem Löß, pedogen überprägtem Löß sowie die Ansprache von genetischen Bodenhorizonten wie CB-, BC-, Ah-, Bw-, B- und Bt-Horizonten.

Die Löß-Paläoboden-Abfolge der Brunhes-Epoche in Karamaydan/Tadjikistan ist noch wesentlich detaillierter gegliedert als die entsprechende Abfolge in Luochuan/China oder von mitteleuropäischen Lößprofilen mit Ausnahme der Würm-Kalkzeit. So entsprechen z.B. Paläoböden in China oder Mitteleuropa komplizierten Pedokomplexen in Karamaydan. Daher wird die Sequenz von Lössen und stärker entwickelten Böden von Karamaydan als Referenzprofil für die Rekonstruktion der mittel- und jungpleistozänen Klimageschichte vorgeschlagen. Die Löß-Boden-Abfolge erlaubt eine sehr gute Korrelation mit der $\partial 18 \mathrm{O}$-Tiefseekurve von BAssiNOT et al. (1994), für die präzise astronomische Zeitangaben abgeleitet wurden. Dadurch wird sogar eine $\mathrm{Zu}-$ ordnung von Böden innerhalb eines Pedokomplexes

*) Anschrift des Verfassers: Prof. Dr. A. Bronger, Geographisches Institut der Universität Kiel, 24098 Kiel des Referenzprofils von Karamaydan zu Substadien der 218 O-Kurve ermöglicht (Abb. 1). - Für den größeren Teil der Matuyama-Epoche zeigt der mittlere und basale Teil des Lößprofils von Chashmanigar (Tadjikistan) mehr gut ausgebildete Paläoböden als entsprechende Abschnitte der Löß-Boden-Abfolgen von Luochuan/ China (Abb. 2) und sehr viel mehr als in Mitteleuropa. Dabei enthält die altpleistozäne Löß-Boden-Sequenz von Chashmanigar mehrere stratigraphische Lücken, belegt durch Ck- bzw. Ckm-Horizonte, deren zugehörige Paläoböden abgetragen sind. Dennoch ermöglicht diese Sequenz mehr paläoklimatische Informationen bezüglich kalt-trockener Lößzeiten und feuchterer Warmzeiten als die bisher bekannten Tiefseekurven.

Für weitere paläoklimatische Interpretationen sind (ton)mineralogische Untersuchungen an den drei Schluff-Fraktionen sowie der Grob- und Mitteltonfraktion $(2-0,2 \mu \mathrm{m})$ und Feintonfraktion $(<0,2 \mu \mathrm{m})$ von Paläoböden mit ihrem jeweiligen Ausgangssubstrat, dazu dem Vergleich des holozänen klimaphytomorphen Bodens notwendig, um Auskunft über Art und Ausmaß der Verwitterung und Tonmineralbildung zu erhalten. Die Ergebnisse zeigen, daß Art und Ausmaß der pedogenen Tonmineralbildung in den Paläoböden der Brunhes-Epoche und den allermeisten Böden der Matuyama-Epoche in Chashmanigar nicht wesentlich von der der holozänen Böden abweicht. Daraus darf gefolgert werden, daß die Klimate der Interglaziale, repräsentiert durch B- oder Bt-Horizonte der jung-, mittel- und altpleistozänen Paläoböden, klimatisch ähnlich dem des Holozäns gewesen sind.

\section{[Loess-Paleosol sequences in Central Asia - towards a Quaternary paleoclimatic history on a global scale]}

Abstract: Recent small climatic fluctuations on a $10^{2}-10^{3}$ year time scale can be correlated worldwide for example by distinct moraines dated to about $1850 \mathrm{AD}$ in similar positions below the present day glaciers in the southeastern Canadian Rockies, in the European Alps and in the Tian-Shan near Urumqi, China. These moraines result from glacier advances caused by a decline of mean annual temperature of only about $1{ }^{\circ} \mathrm{C}$. This suggests that major climatic changes on at least a $10^{5}$ year scale (glacial-interglacial cycles) and probably a $10^{4}$ year scale (the approximate length of an interglacial) must be of similar ages throughout the temperate climatic belt of the Northern Hemisphere. This concept is important for continental pedostratigraphical correlations; especially when loess-paleosol sequences correspond with time equivalent parts of the deep-sea oxy- 
gen isotope record in the Brunhes epoch we are in all probability close towards a continental or even a global chronostratigraphical correlation between loess-paleosol sequences in different continents. This allows the reconstruction of a quaternary climatic history especially of the Brunhes epoch in the temperate climatic belt of the northern hemisphere.

Detailed knowledge of the genesis of paleosols is needed to establish loess-paleosol stratigraphies that can be used for paleoclimatic reconstruction. Most p: leosols, however, are truncated and largely ıecalcifieci by carbonate derived from overlying loess. ": Aicromorphological studies allow primary and secondary carbonates to be distinguished and provide unequivocal evidence of clay illuviation. This enables the separation of typical loess, weakly weathered loess and the recognition of different genetic soil horizons as $\mathrm{CB}, \mathrm{BC}, \mathrm{Ah}, \mathrm{Bw}, \mathrm{B}$ and Bt horizons. A comparison of the loess-paleosol sequence of the Karamaydan section/Tadjikistan with the upper part of the Luochuan section/China clearly indicates that for the Brunhes chron the sequence at Karamaydan, Tadjikistan, is more detailed than the corresponding section in Luochuan, China and even more than in Central Europe except for the last glaciation. Single paleosols at Luochuan correspond to pedocomplexes at Karamaydan such as S3 soil to PK III and the S4 soil to PK IV; both PKs developed in a time period much longer than one interglacial (about $10^{4}$ years). Some pedocomplexes at Luochuan have more detailed successions at Karamaydan; e.g. the S5-complex in Luochuan, which corresponds with PKs VI and V. The loess-paleosol sequence in Karamaydan, therefore, should be regarded as a key sequence for reconstructing the climatic history of the Brunhes epoch. The good correlation with the deep-sea oxygen isotope record of BASSINOT et al (1994), which includes the development of an accurate astronomical time scale, allows a detailed chronostratigraphical subdivision of the loess-paleosol sequence in Karamaydan for the Brunhes epoch. Also, for most of the Matuyama epoch the central and lower parts of the sequence at Chashmanigar, Tadjikistan, show more pronounced paleosols than the equivalent parts of the sequences at Luochuan and much more than in Central Europe, although some $\mathrm{Ck}$ or $\mathrm{Ckm}$ horizons indicate the presence of hiatuses or lost parts of the Chashmanigar section. Nevertheless it provides more paleoclimatic information regarding cold arid stages represented by loesses and warm humid stages (interglacials) represented by paleosols, than even the deep-sea cores known so far.

To reconstruct paleoclimates the primary and secondary minerals of the silt and clay fractions must be determined separately to evaluate the type and intensity of mineral weathering and clay mineral formation. This shows that there is little difference in the type and amount of pedogenic clay mineral formation between the Holocene soils and the paleosols of the Brunhes epoch at Karamaydan and of most of the paleosols in the Matyama epoch at Chashmanigar. This suggests that the interglacial climates represented by the $\mathrm{B}$ or $\mathrm{Bt}$ horizons of the buried paleosols of young, middle and old Pleistocene age were similar to that of the Holocene.

\section{Einführung und Zielsetzung}

Die Geländeposition der Seitenmoränen um 1850 unterhalb der zugehörenden heutigen Gletscher in vielen Talschlüssen der Alpen ist überraschend ähnlich den 1850er Moränen etwa am AthabaskaGletscher in den südöstlichen kanadischen Rockies oder im Tien-shan oberhalb von Urumqi/Xinjiang, China. Diese Moränen sind das Ergebnis von Gletschervorstößen, verursacht durch eine Abnahme der Jahresmitteltemperatur von 0,4-0,8 ${ }^{\circ} \mathrm{C}$ für die Alpen (MaISCH 1995: 687). Daraus darf gefolgert werden, daß wesentlich größere quartäre Klimaschwankungen im Bereich von $10^{5}$ Jahren (Glazial-Interglazial-Zyklus) und aller Wahrscheinlichkeit nach auch im Bereich von $10^{4}$ Jahren (etwa der Länge eines Interglazials, s. Kap. 3, 4.2) weitestgehend gleichzeitig, jedenfalls im gemäßigten Klimabereich der Nordhemisphäre stattgefunden haben müssen.

Diese Hypothese oder dieses Konzept ist wichtig für kontinentale pedostratigraphische Korrelationen: wenn eine paläomagnetisch „datierte“ LößPaläoboden-Abfolge der Brunhes-Epoche möglichst an einer Stelle aufgeschlossen ist, die mit der $\delta^{18} \mathrm{O}$-Tiefseekurve des gleichen Zeitabschnitts in gute Übereinstimmung gebracht werden kann, so müßte man Löß-Paläoboden-Sequenzen des Mittel- und Jungpleistozäns selbst in verschiedenen Kontinenten miteinander korrelieren, insbesondere pedostratigraphische Lücken feststellen können. Das würde dann - bei Anwendung geeigneter paläopedologischen Untersuchungsmethoden (Kap. 2) - die Rekonstruktion einer Klimageschichte der letzten 800.000 Jahre für den gemäßigten Klimabereich der Nordhemisphäre erlauben.

Solche Löß-Paläoboden-Sequenzen, die sich eng mit der $\delta 18 \mathrm{O}-$ Tiefseekurve der Brunhes-Epoche (BAssinot et al. 1994) korrelieren lassen, sind in der Tadjikischen Depression aufgeschlossen. Für die Matuyama-Epoche sind dort Lößprofile durch fossile Böden so detailliert gegliedert wie kaum irgendwo sonst, insbesondere nicht in Mitteleuropa (Kap. 4.3); jedoch fehlt im Unterschied zu entsprechenden Sequenzen des chinesischen Lößplateaus bisher eine ausreichende paläomagnetische „Datierung“ (s. Kap. 4.1).

Lößprofile in Zentralasien insbesondere in Tadjikistan sind früher aus geologisch-stratigraphischer Sicht beschrieben worden, insbesondere von Dodonov (z. B. 1979, 1984, 1986, 1991), und Lazarenko (z. B. 1984). Aber selbst die Ck- z. T. Ckm-Horizonte mit ausgeprägten Kalkkonkretionen („Lößkindl"), die für viele Paläoböden in den 
Profilen von Karamaydan und besonders Chashmanigar (Kap. 3: Abb. 1 u. 2) ein charakteristisches Merkmal sind und belegen, daß die Böden autochthon sind, wurden von den genannten $\mathrm{Au}-$ toren nicht erwähnt. Erste Ergebnisse zur Genese der Paläoböden - eine von mehreren Voraussetzungen für ihre paläoklimatische Interpretation (s. Kap. 2) - wurden kürzlich vorgestellt (BRONGER et al. 1995). Durch weitere Untersuchungen insbesondere zur Verwitterungsintensität jung-, mittel- und altpleistozäner Böden und ihr Vergleich mit denen des Holozäns (Kap. 3) können Schlußfolgerungen zu einer quartären Klimageschichte abgeleitet werden. Die Löß-Paläoböden-Sequenzen dieses Raumes werden dann - unter Zuhilfenahme der o. g. $\delta 18 \mathrm{O}-$ Tiefseekurve - denen Zentralchinas und Mitteleuropas (Kap. 4) gegenüberstellt.

\section{Methodische Aspekte: Möglichkeiten und Grenzen der Paläopedologie. - Das Untersuchungsgebiet}

Die bodenbildenden Faktoren Klima, Vegetation (mit Fauna), Relief (mit Zuschußwasser), Ausgangssubstrat und Zeit lösen bodenbildende Prozesse aus, die sich in den Merkmalen eines Bodens niederschlagen. Diese „Kausalkette der Pedogenese" (SCHRÖDER 1992) läßt sich auch umkehren: aus Bodenmerkmalen kann man auf bodenbildende Prozesse schließen, damit auf die Bodengenese. Diese erlaubt bei klimaphytomorphen Böden Rückschlüsse auf den bodenbildenden Faktor Klima und, damit eng verknüpft unter Umständen auf die Vegetationsformation, unter der sich dt: Boden gebildet hat (s. u.). Klimaphytomorphe Böden liegen dann vor, wenn - wie bei Lößböden - das Ausgangssubstrat stets etwa das gleiche ist und sich die Böden in grundwasserfernen Plakorlagen gebildet haben. Es bleibt die Frage, ob sich ein Paläoboden in ähnlicher zeitlicher Dimension ( $10^{4}$ Jahre) gebildet hat (vgl. Kap. 4.2) wie der Boden des Holozäns.

Für eine Löß-Boden-Stratigraphie und insbesondere für paläoklimatische Rückschlüsse ist es also einerseits notwendig, die Genese eines begrabenen Paläobodens über seine Merkmale zu erschließen. Das ist deswegen schwierig, weil einmal die meisten Paläoböden erodiert sind: fast immer ist der A-Horizont, oft noch ein größerer Teil des B-Horizontes vor allem in einer Phase mit Solifluktion am Übergang einer Warmzeit zur folgenden Lößkaltzeit abgetragen. Damit fehlen wesentliche diagnostische Merkmale etwa zur Ansprache eines Tonanreicherungshorizontes (,ar- gillic horizon") oder eines „mollic“ Horizontes für die Zuordnung eines Bodens in die Ordnung der Alfisols bzw. Mollisols der "Soil Taxonomy“" (SOILSURVEY STAFF 1996) oder der Luvisols bzw. Phaeozems oder Chernozems in die „World Reference Base" WRB (ISSS-ISRIC-FAO 1994). Zum anderen sind in Lößgebieten die begrabenen Böden aus dem hangenden Löß mehr oder weniger sekundär aufgekalkt, so daß im Unterschied zu heutigen oder holozänen Oberflächenböden pH- oder Basensättigungswerte keinen Sinn machen. Deshalb ist die Bodenmikromorphologie von besonderer Wichtigkeit, weil sie z. B. die Unterscheidung von primären und sekundären Calciten erlaubt (Beispiele in BRONGER 1976, Taf. I-VI). Diese genetische Trennung zwischen primären und sekundären Calciten ermöglicht z. B. die Unterscheidung von Degradierten Tschernosemen jetzt Phaeozemen in der WRB und den Tschernosemen i.e.S., die noch primäre Calcite im unteren Teil des Bodens enthalten. Die Phaeozeme aus Löß sind klimaphytomorphe Böden der (feuchteren) Waldsteppe, während die Tschernoseme i.e.S. die klimaphytomorphen Böden der (trockeneren) Langgrassteppe sind. - Andererseits ermöglicht die Mikromorphologie den Prozeß der Tonverlagerung zweifelsfrei zu erkennen. Dieser Prozeß läuft in Lößböden in Plakorlage (s.o.) im pH-Bereich von 4,5-6 (0,1 M KCl oder $\left.\mathrm{CaCl}_{2}\right)$ ab. Bei Abwesenheit von $\mathrm{Na}+$-Ionen findet dieser Prozeß im gemäßigten Klimabereich unter Wald, aber nicht mehr unter einer Steppe i.e.S. statt. Dieser kausale Zusammenhang wurde in vielen holozänen Lößböden Ostmittel- und Osteuropas sowie in den westlichen Central Lowlands und Great Plains der USA gefunden ( $\mathrm{z}$. B. Bronger 1976: 35-106; 1991).

Für weitere paläoklimatische Interpretationen sind mineralogische Untersuchungen notwendig, um Auskunft über Art und Ausmaß der Verwitterung und Tonmineralbildung zu erhalten.

Hierfür wurden jeweils die drei Schluff- und die beiden Tonfraktionen von zwei holozänen Böden innerhalb einer Toposequenz, dazu von sieben jung- und mittelpleistozänen Paläoboden bzw. Pedokomplexen sowie von neun altpleistozänen Paläoböden quantitativ gewonnen. Der Mineralbestand wurde polarisationsoptisch an $\geq 300$ Körnern in der Fraktion 63-20 $\mu \mathrm{m}$ und phasenoptisch an 700-1000 Körnern in den Fraktionen 20-6 bzw. 6-2 $\mu \mathrm{m}$ bestimmt - der Sandanteil fehlt in den tadjikischen Lössen oder ist $\leq 1 \%$. Die Prozentanteile der Minerale bzw. Mineralgruppen in jeder Fraktion (Kornzahlprozente) wurden dann 


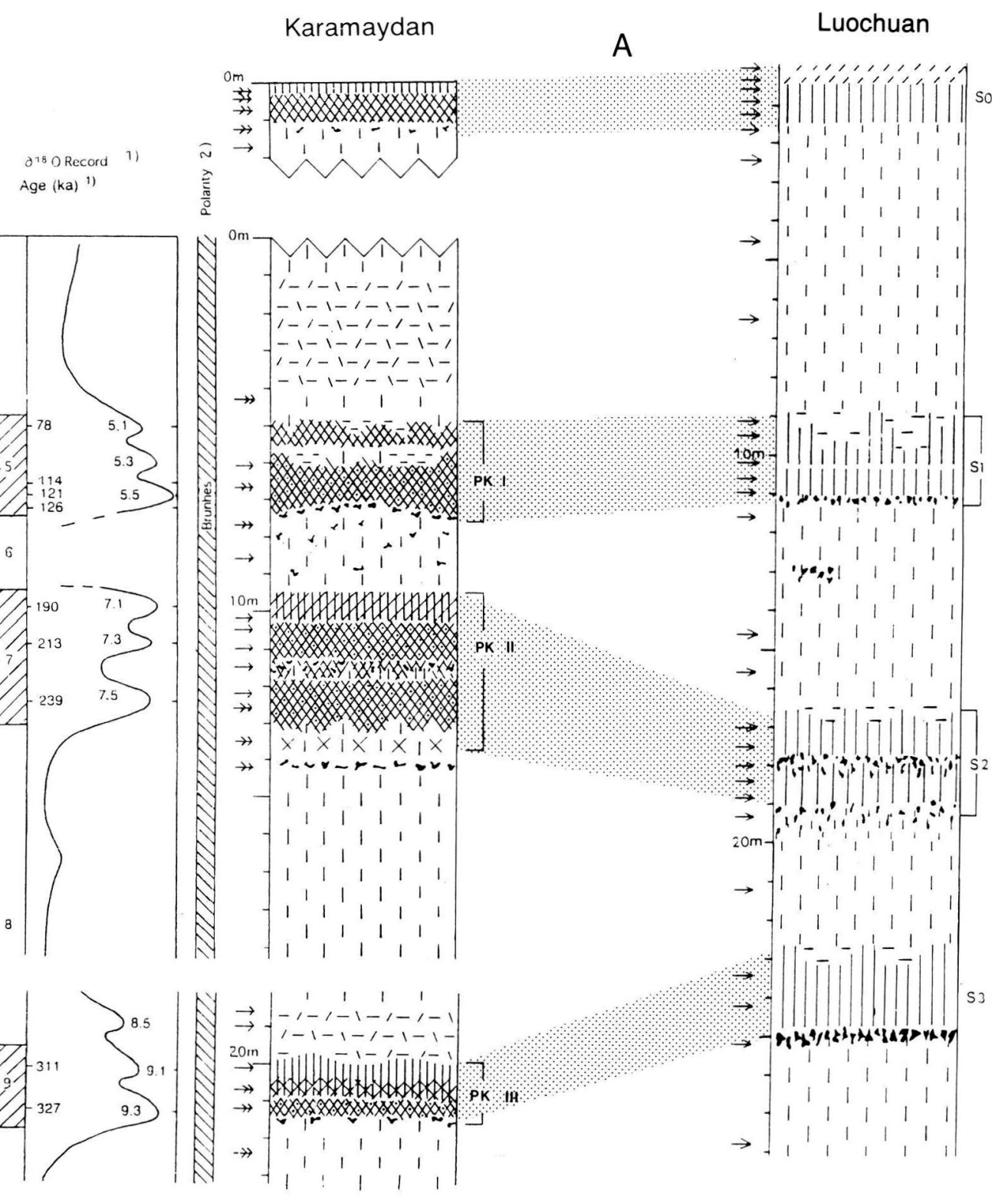

$\begin{array}{cl}\text { WS - } 1-\text { WL - } 3 & \text { pedostratigraphische Einheiten } \\ \text { S } 1-S 14 & \text { nach LIU et al. } 1985\end{array}$

1) nach BASSINOT et al. 1994, angepaßt

2) nach PENKOV \& GAMOV 1980, FORSTER and HELLER 1994

3) nach HELLER \& LIU 1982, 1984

Abb. 1: Genese der Paläoböden und pedostratigraphische Korrelation der Lößprofile von Karamaydan, A: PK I - PK III mit S1-S3, B: PK IV - PK IX mit S4-S7

Fig. 1: Genesis of paleosols and pedostratigraphic correlation between the sections of Karamaydan, Central Asia A: PK I - PK III with S1-S3, B: PK IV - PK IX with S4-S7 


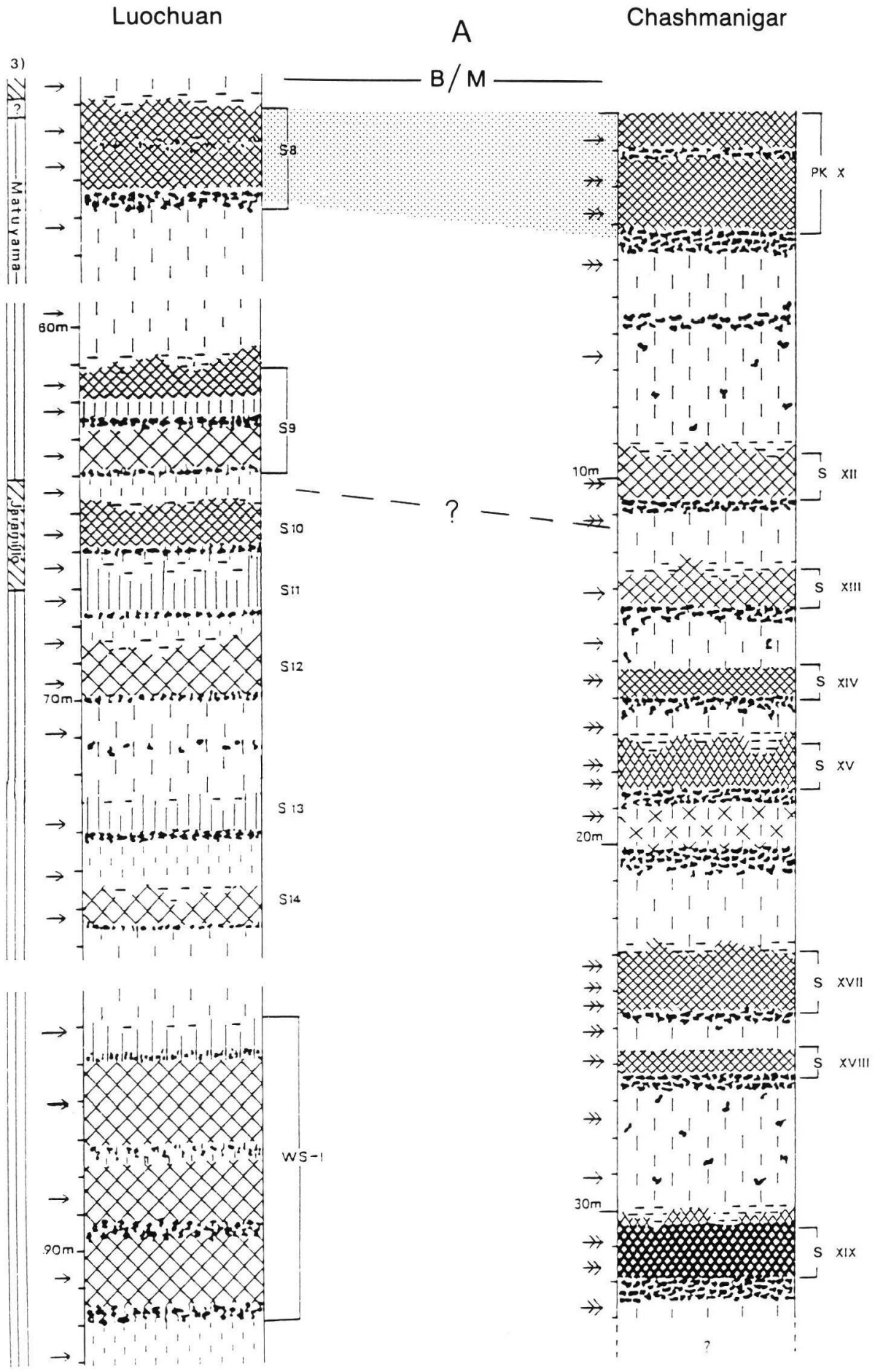

Zentralasien und Luochuan, Ostasien.

and Luochuan, East Asia. 
Karamaydan
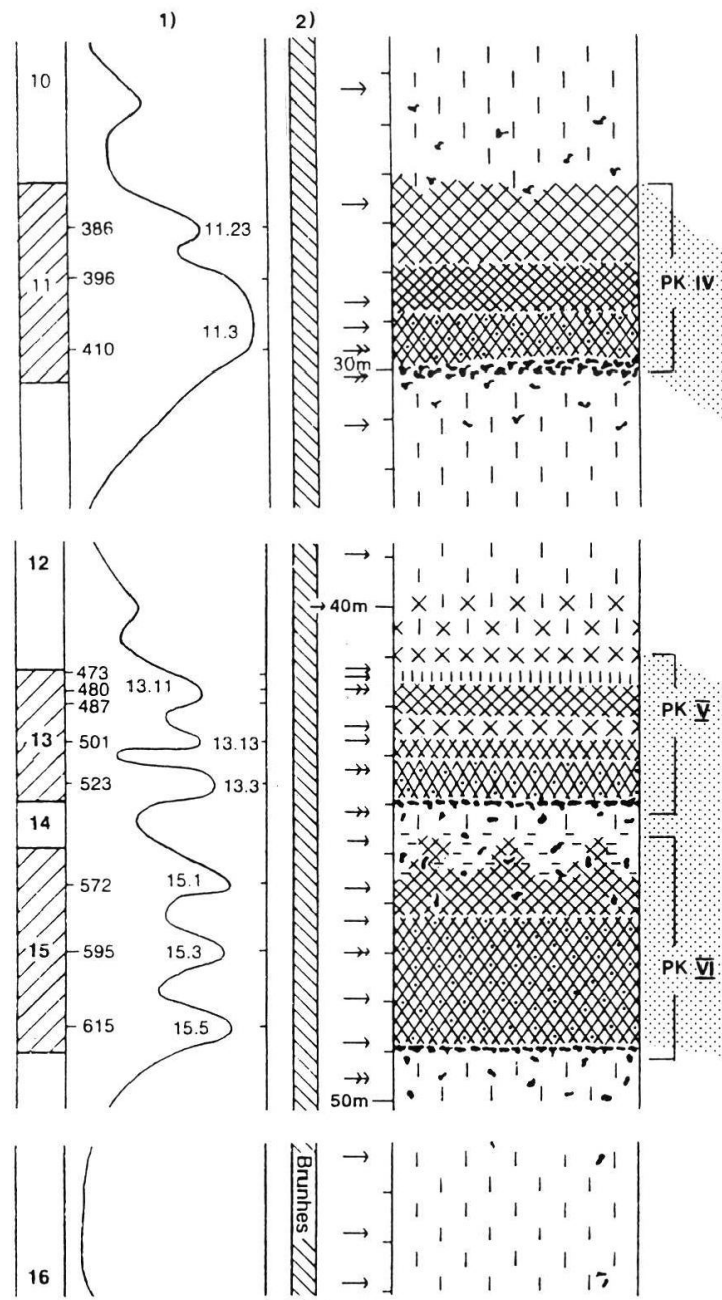

Luochuan

B
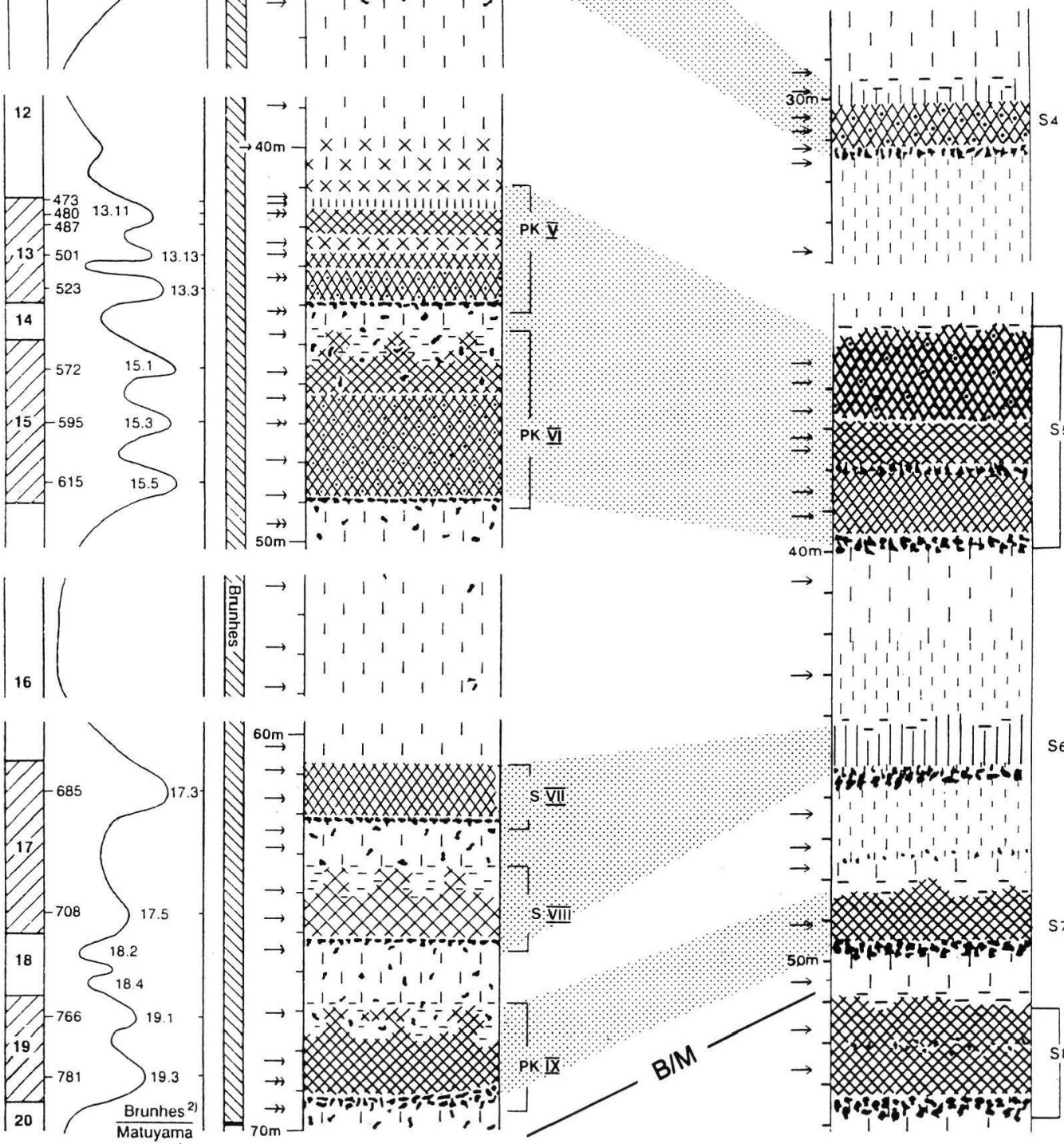

Hotentrainthatp:

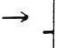

$1 \quad 1 \quad 1$

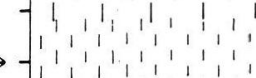

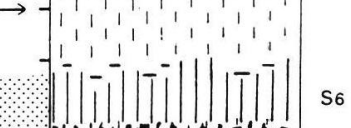

Abb. 2: Genese der Paläoböden und Vergleich der altpleistozänen Abschnitte der Lößprofile von A: mittlere Teile beider Profile, B: untere Teile beider Profile

Fig. 2: Genesis of paleosols and comparison with Lower Pleistocene sections (Matayama epoch) of the loess A: central parts, B: lower parts of both sections 


\section{Luochuan}
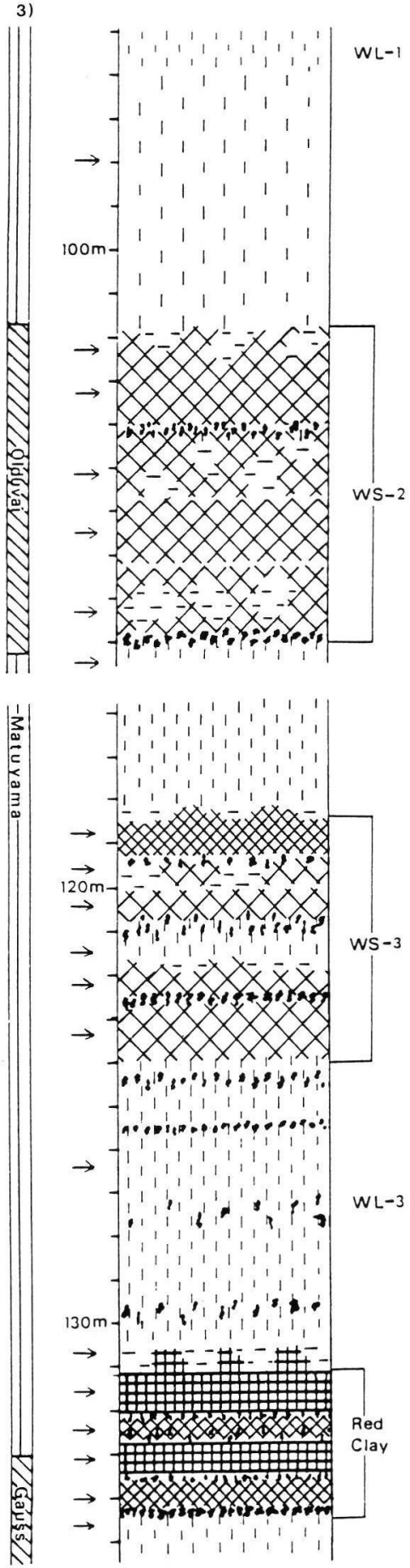

\section{Chashmanigar}

B

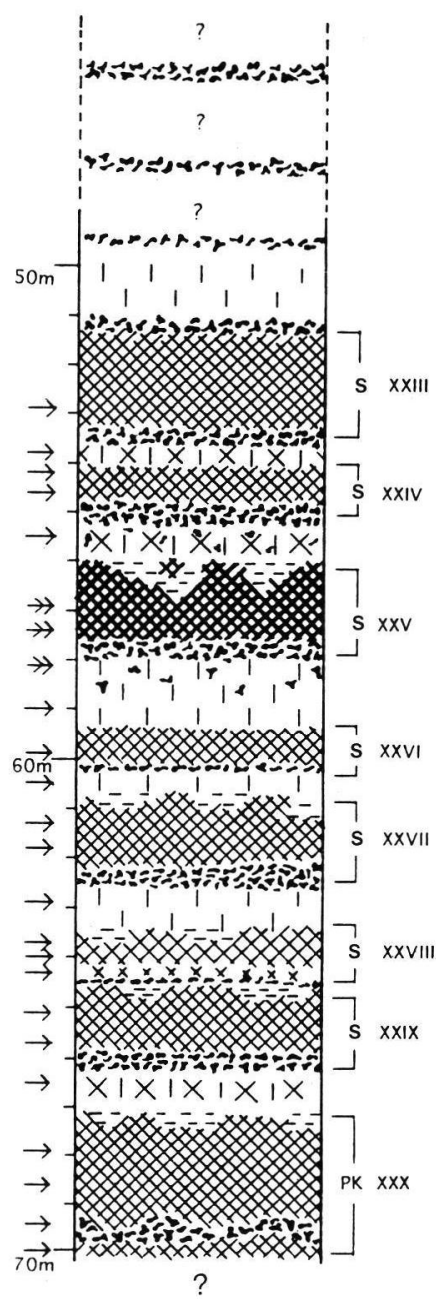

Legende zu den Abb. 1 und 2:

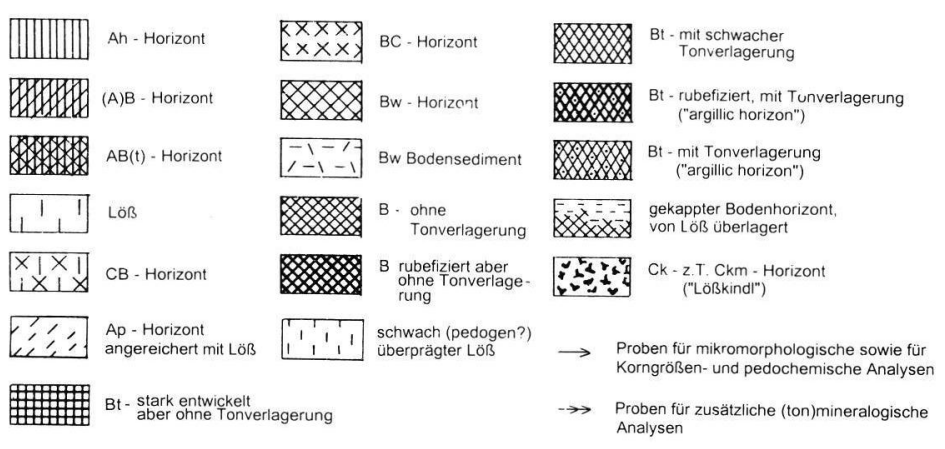

Chashmanigar, Zentralasien und Luochuan, Ostasien.

exposures of Chashmanigar, Central Asia and Luochuan, East Asia. 
mit den Korngrößenanteilen multipliziert, um zu Gewichtsprozenten jeder Mineralgruppe zu kommen. Diese sind in den Abb. 3-6, auf der jeweils linken Seite dargestellt. - Für die Analyse der silicatischen Tonminerale wurde die Fraktion 2-0,2 $\mu \mathrm{m}$ und die pedogenetisch besonders wichtige Feintonfraktion $<0,2 \mu \mathrm{m}$ ebenfalls quantitativ gewonnen. Die Zusammensetzung der Tonteilfraktionen wurde röntgendiffraktometrisch nach Aufweitungs- und Kontraktionstests auf der Basis der Summe der Flächen ausgewählter Peaks abgeschätzt. Bei der röntgenographischen Untersuchung nur der Gesamtfraktion würden die Interferenzen der Feintonfraktion weitgehend unterdrückt. Die so gewonnenen Kornzahlprozente der Tonminerale bzw. Tonmineralgruppen wurden dann mit dem Gewichtsanteil der jeweiligen Tonteilfraktion multipliziert. Diese Gewichtsprozente, dargestellt auf der jeweils rechten Seite der Abb.3-6, sind folglich nur relativ quantitative Abschätzungen im Unterschied zu den Gewichtsprozenten der Fraktionen $>2 \mu \mathrm{m}$ (näheres s. Bronger \& KAlK \& SCHRÖDER 1976, BRONGER \& HEINKELE 1990).

Eine Auskunft über Art und Ausmaß der Verwitterung der Primärminerale $>2 \mu \mathrm{m}$ und pedogener Bildung der Tonminerale ist aber nur dann möglich, wenn das bodenbildende Substrat petrographisch homogen ist. Zur Überprüfung eignet sich Quarz als in Lössen in ausreichenden Mengen vorkommendes, mindestens im gemäßigten Klimabereich verwitterungsstabiles sog. Indexmineral (BARSHAD, 1967). Eine Gewichtskonstanz des Quarzes in den verschiedenen Bodenhorizonten (einschließlich des C-Horizontes) ist kein Beweis, aber ein guter Beleg für eine ursprüngliche petrographische Homogenität.

Ausgangspunkt vor allem auch der mikromorphologischen und tonmineralogischen Untersuchungen muß dabei der klimaphytomorphe holozäne Boden sein. Hier ist die Konstellation bodenbildender Faktoren bekannt, wobei die Rekonstruktion der potentiellen natürlichen Vegetation nicht immer leicht ist.

Das Klima des Untersuchungsgebietes ist charakterisiert durch kühle, feuchte Winter und trockene, recht warme Sommer. Der deshalb „xeric“ Bodenwasserhaushalt (SOIL SURVEY STAFF 1975: 57) ist gekennzeichnet durch einen Wasserüberschuß im Winter. Dem Wasserdefizit im späten Sommer geht deshalb eine etwa dreimonatige Periode des Wasserverbrauchs infolge der hohen Nutzwasserkapazität der Lößböden voraus. Im Herbst wird der Boden mit Wasser wieder aufge- füllt (s. Bronger et al. 1995, Abb. 2). - In den letzten ca. 100 Jahren ist infolge enormen Bevölkerungswachstums und daraus folgend starker Überweidung besonders durch Schafe und Ziegen die Landschaft zu einer (z. T. Kameldorn-) Steppe degradiert. Deshalb wurde sogar die frühere Existenz von Wäldern zwischen 1000$2000 \mathrm{~m}$ ü. NN in Zweifel gezogen (Lomov 1985). Die Bodenerosion hat sehr große Ausmaße angenommen; so wurden auch die beiden Lößprofile von Karamaydan und Chashmanigar im Frühjahr 1992 durch Lößmuren teilweise verschüttet. Dennoch gelang es, die potentielle natürliche Vegetation zu rekonstruieren (Zapriagaeva 1964, 1968; STANINKOVITCH 1968): danach bestand der Wald südlich des Tien-shan und westlich des Pamir in 1000-2200 (2400) m ü. NN aus Hasel (Acerturkestanicüm), Platane (Plantanus orientalis), Esche (Fraxinus potomolina), Walnuß (Juglans regia) und weiteren Wildobstbaumarten wie Mandelbaum (Amygdalus communis, A. bucharica) und verschiedenen Arten des Zürgelbaumes (Celtis), Birnbaum (Pyrus), Weißdorn (Crataegus) etc. In mehreren Gebieten zwischen 1700-2300 m ü. NN war der Wachholder (Juniperus seravschanica) verbreitet.

\section{Ergebnisse und Interpretation}

Die beiden hier näher untersuchten holozänen klimaphytomorphen Böden des Untersuchungsgebietes haben einen $27 \mathrm{~cm}$ mächtigen dunkelgrauen Ah-Horizont mit mikromorphologisch aggregatreichem Feinschwammgefüge, damit einen "mollic" Horizont. Die zugehörenden BtHorizonte, $105 \mathrm{~cm}$ bzw. $80 \mathrm{~cm}$ mächtig, haben eine scharfe Grenze zum weißlich-gelben Ck-Horizont, was typisch für Waldböden, nicht für Steppenböden ist. Die pH-Werte (in $1 \mathrm{M} \mathrm{KCl}$ ) liegen bereits bei $\geq 4,9$. Die Unterböden zeigen auch infolge noch hoher biologischer Aktivität nur sehr geringe Anteile an orientiertem Feintonplasma als Zeichen für eine Tonverlagerung, die zu schwach ist, um die Horizonte als „argillic“ (SOIL SURVEY STAFF 1975, 1996) bzw. „argic“ (ISSS-ISRIC-FAO 1984) einzuordnen. Insgesamt sind die untersuchten holozänen klimaphytomorphen Böden infolge ihres „xeric" Bodenwasserhaushaltes als Typic Haploxerolls in der Soil Taxonomy bzw. als Haplic Phaeozems in der WRB anzusprechen.

Die Abgrenzung der Horizonte innerhalb der fossilen Böden bzw. Pedokomplexe (PK's) wurden im Gelände, die genetische Ansprache der Horizonte bzw. der Ausgangssubstrate mikromorphologisch anhand von Dünnschliffen von 160 orien- 
tierten Proben vorgenommen. Die Ergebnisse sind im zentralen Teil der Abb. $1 \mathrm{~A}+\mathrm{B}$ für die jung- und mittelpleistozänen Abschnitte des Lößprofils von Karamaydan und dem rechten Teil der Abb. 2 A + B für den altpleistozänen Abschnitt des Lößprofils von Chashmanigar zusammengefaßt. Eine Diskordanz unterhalb des PK X in Karamaydan wie auch in Chashmanigar sichert die stratigraphische Korrelation zwischen beiden Profilen. Die Grenze vom Mittel- zum Altpleistozän wird hier wie meist üblich an die Brunhes/Matuyama-Grenze gelegt, die im Profil von Karamaydan im Löß zwischen PK IX und PK X gefunden wurde (Penkov \& Gamov 1977, 1980; Forster \& Heller 1994; vgl. Kap. 4.1). Die Plio-/Pleistozängrenze legen wir an die Matuyama/Gauss-Grenze (vgl. ZAGWIJN 1992), auch wenn die Sedimentation von Löß oder Lößderivaten in Luochuan/China schon etwas früher einsetzte (vgl. Bronger \& HeINKELE 1989 b, Fig. 2). Hier können aus Raumgründen nur einige Punkte zur genetischen Ansprache von Lössen und Bodenhorizonten hervorgehoben werden (zu Details vgl. Bronger \& Winter \& HeINKELE 1998):

Typischer Löß enthält sehr zahlreiche primäre klastische Calcite besonders in der Mittel- und Grobschluff-Fraktion, regellos in der Matrix verteilt, neben sekundären Calciten, die vor allem in der Feinschluff-Fraktion feinverteilt in der Matrix oder in Hohlräumen angereichert sind. Sie sind ein Zeichen stärkerer Bicarbonat-Metabolik in einem nicht ganz so trockenen Klima. Die typischen Lösse besonders im mittleren und oberen Teil des Profils von Karamaydan haben einen sehr hohen Anteil von primären Calciten, was recht trockenes Klima anzeigt. Dieser Löß findet sich in allen Teilen des Profils von Karamaydan und im Profil von Chashmanigar bis unterhalb des Paläobodens $\mathrm{S}$ (PK?) XXVII.- Schwach pedogen überprägter Löß enthält in Teilen ein schwach ausgebildetes Mikroaggregatgefüge, andererseits noch primäre Calcite. Dieser Lößtyp ist recht selten in Karamaydan und Chashmanigar, dagegen häufig im altpleistozänen „Wusheng"-Löß in Luochuan/China (vgl. Abb. 2, linke Seite; s. Kap. 4.1), wie auch im mittelpleistozänen „Lishi“-Löß unterhalb des Paläobodens S4 sowie oberhalb und unterhalb des Paläobodens S6 des genannten Lößprofils (s. Abb. 1 B, rechte Seite).

Unter den Bodenhorizonten enthalten CB-Horizonte noch primäre Calcite: bei ihnen überwiegen die Merkmale des Lösses gegenüber B-Horizonten. Sie sind meistens ein Gemisch von Löß i. w. S. und B-Horizont-Material z. B. durch Soli- fluktion. BC-Horizonte sind Übergänge zwischen B- und C- bzw. Ck-Horizonten und enthalten keine primären Calcite mehr. Das gilt natürlich erst recht für Bt- bzw. B-Horizonte, die sich durch die An- oder Abwesenheit von Feintonplasma (,illuviation argillans", BREWER 1964) unterscheiden. Beispiele für Bt-Horizonte „mit geringer (mikromorphologisch sichtbarer) Tonverlagerung" sind die der beiden holozänen Böden (s. o.); solche BtHorizonte sind aber in den genannten Lößprofilen nicht selten (s. Abb. 1,2). Nur wenige Bt-Horizonte haben $\geq 1 \%$,illuviation argillians", welche sie diagnostisch als „argillic horizons“ ausweisen. Dazu gehören mehrere innerhalb der Pedokomplexe I-VI in Karamaydan, in Chashmanigar nur der BtHorizont des S XV (s. Figs. 1, 5 u. 6 in Bronger \& Winter \& Heinkele 1998), sowie der Bt-Horizont des S4 und der obere Bt-Horizont des S5-Pedokomplexes in Luochuan/China (s. Kap. 4.1). BwHorizonte sind ein Übergangsstadium von schwach überprägtem Löß (s.o.) und B-Horizonten ohne Tonverlagerung. Sie haben in Teilen ein schwach ausgebildetes Aggregatgefüge, sind aber frei von primären Calciten. Wenn sie mächtiger sind wie der obere Bw-Horizont des PK IV oder der S VIII in Karamaydan oder sehr mächtig sind und sogar Lößkindl-Horizonte enthalten wie in den Pedokomplexen WS-1 bis WS-3 des „Wuscheng"-Löß in Luochuan (s. Abb. 2, rechte Seite; vgl. Kap. 4.1), so sind sie wahrscheinlich synsedimentär durch alternierende Lößakkumulation und Bodenbildung entstanden. Ah-Horizonte sind mikromorphologisch charakterisiert durch ein aggregatreiches Feinschwammgefüge infolge hoher biologischer Aktivität. Sie sind mit Ausnahme des oberen Teils des PK III und PK V stets abgetragen, so daß eine Klassifikation als Xerolls oder Xeralfs bzw. Phaeozems oder Luvisols nicht möglich ist.

In den Lößprofilen von Karamaydan und in kleineren Teilen auch in Chashmanigar, aber auch in Luochuan/China (Kap. 4.1) werden die Paläoböden z.T. zu Pedokomplexen zusammengefaßt. Für zentralasiatische Lößprofile wurde das auch schon von LAZARENKo (1984) und Dodonov (z. B. 1991) gemacht, aber nicht immer in der gleichen Weise wie hier. Dieses Konzept wurde wohl zuerst in der Tschechoslowakei angewandt (z. B. SMOLIKOvA 1967, 1971, 1990). Pedokomplexe enthalten mindestens zwei Paläoböden bzw. Bodenhorizonte von z. T. erodierten Paläoböden (vgl. Kap. 2), die meistens, aber nicht immer durch ein sehr geringmächtiges, pedogen nicht oder kaum überprägtes Substrat und/oder eine Lößkindl-Lage getrennt sind (s. Abb. 1,2). Pedokomplexe treten beson- 
ders in Karamaydan auf (PK I - PK VI und wahrscheinlich PK IX s. Abb. 1 B), im altpleistozänen Abschnitt von Chashmanigar mindestens als PK X und PK XXX. Jedoch können zukünftige Untersuchungen ergeben, daß weitere Paläoböden polygenetische Pedokomplexe sind: so sind mindestens der S XIX und der S XXIII, obwohl erodiert, für monogenetische B-Horizonte zu mächtig (s. Abb. 2). Ein weiteres Beispiel, der „F6“ von Stari Slankamen (Abb. 6) wird in Kap. 4.3 vorgestellt. In Luochuan/China (Kap. 4.1) sind die stratigraphischen Einheiten S1, S2, S5, S8 und S9 Pedokomplexe, dazu die bereits genannten Einheiten WS1 bis WS3 (s. Abb. 1,2); die Bezeichnungen richten sich dabei nach Liu, T.S. et al. (1985).

Alle Paläoböden haben ausgeprägte Ck- oder sogar Ckm-Horizonte mit dichten Lößkindl-Lagen. Letztere sind besonders ausgeprägt im altpleistozänen Abschnitt von Chashmanigar (Abb. 2). Im Unterschied zu Lössen oder Paläoböden sind mächtige $\mathrm{Ckm}$-Horizonte sehr widerständig gegen Erosion, so daß sie in Aufschlüssen als harte Lagen hervortreten. Sie zeigen Schichtlücken in Löß-Paläoboden-Abfolgen an, wie am Beispiel der altpleistozänen Abfolge in Chashmanigar gezeigt werden kann (Abb. 2). So ist z. B. zwischen dem sehr ausgeprägten $\mathrm{Ckm}$-Horizont an der $\mathrm{Ba}$ sis des PK X und einem ebenfalls markanten Ckm-Horizont $2 \mathrm{~m}$ unterhalb nur noch typischer Löß vorhanden. Aller Wahrscheinlichkeit nach ist der zugehörende Boden vollständig erodiert; deshalb wurde der liegende Boden als S XII bezeichnet. Zwischen dem Ckm-Horizont des S XV und einem weiteren sehr ausgeprägtem Ckm-Horizont ist nur noch CB-Horizontmaterial (s.o.) vorhanden, wahrscheinlich der Rest eines Bodens (S XVI). Zwischen $33 \mathrm{~m}$ und $49 \mathrm{~m}$ in Chashmanigar ist der unzugängliche Steilhang mit etwas Vegetation größtenteils verdeckt. Allerdings sind zwei ausgeprägte Lößkindl-Lagen bzw. Ckm-Horizonte zu sehen, die wahrscheinlich auf zwei weitere Paläoböden (S. XX und S XXI) schließen lassen. Oberhalb des S XXIII liegt unmittelbar ein weiterer Lößkindl-Horizont, darauf nur Löß, so daß ein weiterer Boden (S XXII) abgetragen sein dürfte.

Die Ergebnisse der mineralogisch-tonmineralogischen Untersuchungen an den beiden holozänen Böden (Abb. 3), von sieben ausgewählten jungund mittelpleistozänen Böden bzw. Pedokomplexen (Beispiel in Abb. 4), sowie von neun altpleistozänen Paläoböden sind an anderer Stelle im Detail dargestellt (Bronger \& Winter \& SEDOv 1998, bes. Figs. 2-9). Bezüglich unserer Fragestel- lung können hier einige Ergebnisse folgendermaßen zusammengefaßt werden:

1. Die Gewichtsprozente des Quarzes in den Fraktionen $>2 \mu \mathrm{m}$ steigen von Ck- zum B- oder Bt-Horizont in fast allen Paläoböden der Pedokomplexe nur höchstens sehr geringfügig an, was ein Beleg für die ursprüngliche petrographische Homogenität des bodenbildenden Substrates ist. Nur im S XIV und im S XII in Chashmanigar zeigt der Anstieg des Quarzes von 19,5\% auf $23,5 \%$ bzw. von $21 \%$ auf $26 \%$ auf eine (etwas) größere petrographische Inhomogenität dieser Paläoböden.

2. Die Hauptquellen für die pedogen gebildeten Tonminerale sind Phyllosilikate in den SchluffFraktionen. In der Fraktion 63-20 $\mu \mathrm{m}$, in der Muskovite häufiger sind als die Biotite, zeigen letztere in den B- bzw. Bt-Horizonten sowohl der beiden rezenten Böden wie der Paläoböden besonders in Karamaydan stärkere Verwitterungserscheinungen. Muskovite sind wesentlich verwitterungsstabiler, aber sie tragen aller Wahrscheinlichkeit nach durch mechanische Zerkleinerung zur pedogenen Illitbildung bei. Feldspate spielen als Lieferant für die pedogene Tonbildung eine geringere Rolle.

3. Illite und Vermikulite sind die dominierenden pedogen gebildeten Tonminerale in den B- bzw. Bt-Horizonten sowohl der beiden holozänen Böden wie auch in allen untersuchten jung-, mittelund altpleistozänen Paläoböden bzw. Pedokomplexen mit Ausnahme des S XVII. Dabei kann die Umbildung von Biotiten zu Vermikuliten offenbar rasch vor sich gehen (FANNING et al. 1989). Größere Anteile von Smectiten wurden nur in der Feintonfraktion der B-Horizonte des altpleistozänen S XVII in Chashmanigar gebildet. Recht kleine Anteile von pedogenen Smectiten finden sich in den Bt-Horizonten der Pedokomplexe I-IV in Karamaydan sowie im PK X und S XII in Chashmanigar. In einigen anderen Paläoböden sind die wenigen Smectite offenbar vererbt. - Eine pedogene Kaolinitbildung spielt in keinem der quartären Böden eine Rolle.

4. Für unser Ziel Beiträge zu einer pleistozänen Klimageschichte Zentralasiens zu liefern ist das wichtigste Ergebnis, daß Art und Ausmaß der pedogenen Tonmineralbildung in den beiden holozänen Böden und den jung-, mittel- und altpleistozänen Paläoböden (B- bzw. Bt-Horizonte) im Ganzen recht ähnlich ist. Die Rate der pedogenen Tonbildung insbesondere von Illiten und Vermikuliten in den quartären Böden liegt größtenteils zwischen 10 und $15 \%$. Nur in den unteren Bt-Ho- 

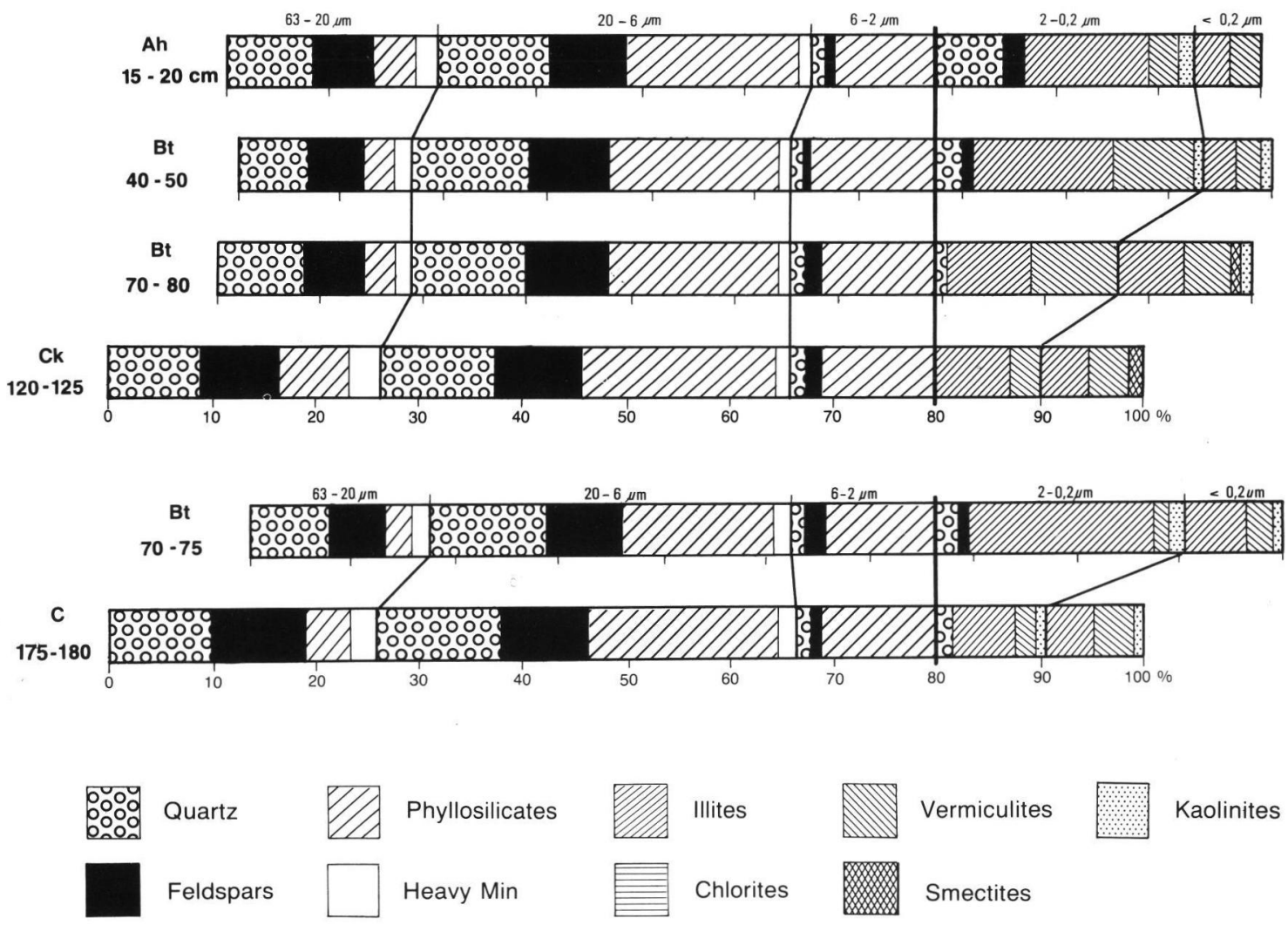

Abb. 3: Mineralogische und tonmineralogische Zusammensetzung von zwei holozänen Böden (Typic Haploxerolls oder Haplic Phaeozems) in der Tadjikischen Depression (1700 mm ü. NN)

Fig. 3: Mineralogical and clay mineralogical composition of two Holocene soils (Typic Haploxerolls or Haplic

Phaeozems) in the Tadjik Depression (1700 m a.s.l).

rizonten der Pedokomplexe I und IV beträgt sie etwa bzw. gut $20 \%$. Selbst in den stärker rubefizierten altpleistozänen Böden S XIX und S XXV, die optisch den Eindruck intensiverer Verwitterung erwecken - mit Farbwerten in der 5 YR Munsell-Skala gegenüber 10-7,5 YR der übrigen Paläoböden - liegt die pedogene Tonbildungsrate im Rahmen der rezenten sowie der übrigen Paläoböden. Aber die Rubefizierung, d. h. ein Anstieg des Hamätit/Geothit-Verhältnisses ist oft (paläo)klimatisch überbewertet worden: relativ höhere Hämatitanteile sind eher abhängig von einem mehr wechselfeuchten Jahresgang mit Trockenzeiten, guter Drainage und geringerem Gehalt an organischer Substanz.

Insgesamt darf aus den hier kurz skizzierten Untersuchungsergebnissen gefolgert werden, daß die Klimate der alt-, mittel- und jungpleistozänen Warmzeiten, repräsentiert durch die stärker entwickelten B-bzw. Bt-Horizonte im Ganzen ähnlich wie im Holozän waren. Einige B- bzw. Bt-Horizonte sind mächtiger als die der beiden holozänen Böden, was auf eine längere Bildungszeit deuten kann. - Unsere Schlußfolgerung, daß die quartären Warmzeiten von der des Holozäns nicht wesentlich verschieden waren, bestätigt aus paläopedologischer Sicht die Schlußfolgerung von SHACKLETON (1987), gewonnen aus d18O-Tiefseekurven, daß der glazialeustatische Meeresspiegelanstieg in keinem der Interglaziale der letzten 2,5 Mill. Jahre höher als einige Meter über dem heutigen lag.

\section{Schlußfolgerungen und Diskussion}

\subsection{Vergleich mit der Löß-Boden-Abfolge in Luochuan/China}

Eine pedostratigraphische Zuordnung der LößPaläoboden-Sequenz von Karamaydan mit der früher paläopedologisch anhand von über 100 Dünnschliffen sowie (ton)mineralogisch untersuchten Sequenz des sehr bekannten Lößprofils von Luochuan (BRonger \& Heinkele 1989a) basiert auf zwei Kontrollpunkten: einmal der Brun- 

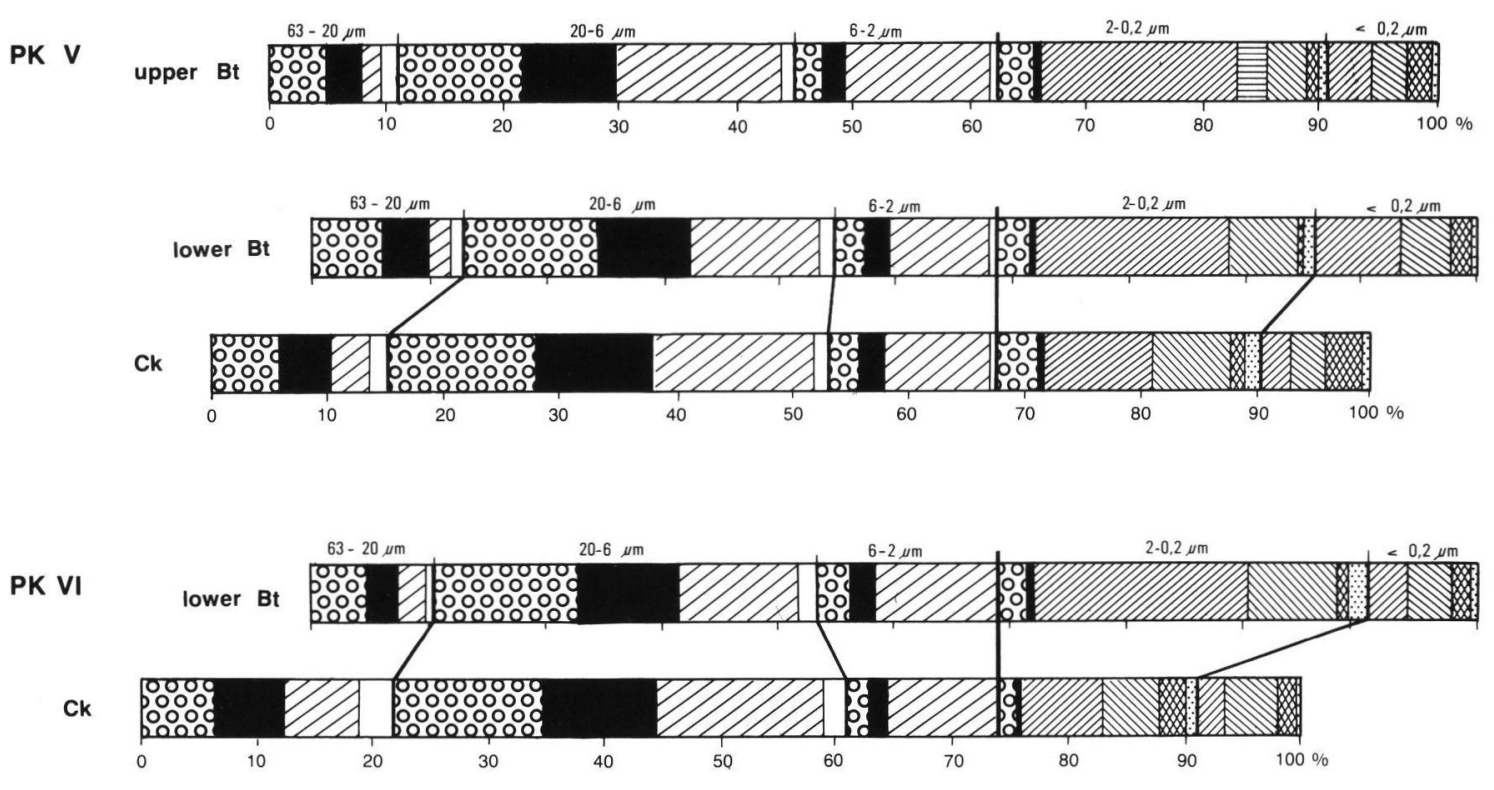

Abb. 4: Mineralogische und tonmineralogische Zusammensetzung einiger Horizonte der Pedokomplexe (PK) V und VI im Lößprofil von Karamaydan, Tadjikistan (1600-1700 m ü. NN).

(vgl. Abb. 1 B, Legende s. Abb. 3)

Fig. 4: Mineralogical and clay mineralogical composition of selected horizons of pedocomplexes (PK) V and VI in the loess profile of Karamaydan, Tadjikistan (1600-1700 m a.s.l)

(see Fig. 1 B, legend see Fig. 3)

hes/Matuyama-Grenze, die in verschiedenen chinesischen Lößprofilen im Löß zwischen den Paläoböden S 7 und S 8 gefunden wurde (zus. fass. Diskussion bei LiU X.M. et al. 1991); zum anderen auf der Korrelation des S1-Pedokomplexes in Luochuan mit dem PK I in Karamaydan (s. Abb. 1), die beide dem Stadium 5 der $\partial^{18} \mathrm{O}$-Tiefseekurve zugeordnet werden (s. Kap. 4.2). Diese Zuordnung wurde in jüngster Zeit durch LumineszenzUntersuchungen bestätigt (FRECHEN \& DODONOV 1998).

Ein Vergleich zwischen den mittel- und jungpleistozänen Löß-Paläoboden-Abfolgen von Karamaydan und Luochuan (Abb. 1) zeigt klar, daß die Sequenz in Karamaydan noch detaillierter gegliedert ist. So entsprechen (scheinbar) monogenetische Böden in Luochuan polygenetischen Pedokomplexen in Karamaydan. Zum Beispiel findet die sehr große Mächtigkeit des Degradierten Tschernosems S 3 (jetzt Phaeozem nach der WRB) vor allem mit seinem anormalen LößkindlHorizont eine Erklärung darin, daß er dem PK III in Karamaydan entspricht: hier wurden zwei Phasen der Bodenbildung durch eine Lößanwehungsphase unterbrochen (vgl. Kap. 4.2). Ebenso entspricht der S 4 in Luochuan dem PK IV in Karamaydan. - Einige einfache aufgebaute Pedokomplexe in Luochuan entsprechen komplizier- ten Pedokomplexen in Karamaydan wie z. B. der S 2-Komplex dem PK II, der sich in einem längeren Zeitraum mit alternierenden Bodenbildungsund Lößanwehungsphasen gebildet hat (näheres s. Kap. 4.2). Der S5-Pedokomplex in Luochuan mit seinen drei Böden entspricht nach unseren Vorstellungen pedostratigraphisch dem PK VI und dem PK V in Karamaydan, dabei der obere rötlicher gefärbte Boden des S5-Komplexes aller Wahrscheinlichkeit nach dem PK V mit seinen drei Bt- bzw. B-Horizonten (näheres s. Kap. 4.2 und 4.3). Der S 6 in Luochuan wird hier (Abb. 1) mit dem S VII und dem S VIII - im Unterschied zu einem früheren Entwurf (BRONGER \& WinTER \& HeInKele 1998) - in Karamaydan parallelisiert, weil der S 6 - wie der S 3, s. o. - für einen Degradierten Tschernosem einen anormal ausgeprägten Lößkindl-Horizont hat.

Zusammengefaßt zeigt das Profil von Karamaydan die detaillierteste Löß-Boden-Abfolge für die Brunhes-Epoche, nicht nur in Zentralasien. Auch weil sich die Abfolge sehr gut mit der $218 \mathrm{O}$-Tiefseekurve von Bassinot et al. (1994) korrelieren läßt (Kap. 4.2) wird vorgeschlagen, die Sequenz von Lössen und stärker entwickelten Böden von Karamaydan als Referenzprofil für die Rekonstruktion der mittel- und jungpleistozänen Klimageschichte anzusehen. Lediglich für die Abschnit- 
te jünger als Eem müssen andere Löß-Boden-Abfolgen herangezogen werden (vgl. Kap. 4.3).

Für die Matuyama-Epoche gibt es für das Lößprofil von Luochuan einen paläomagnetisch „datierten" chronostratigraphischen Rahmen (HELler \& LIu, T. S. 1982, 1984; s. Abb. 2, linke Seite). In Chashmanigar reichte eine etwa $90 \mathrm{~m}$ mächtige, seit 1992 verschüttete altpleistozäne Löß-BodenAbfolge anscheinend bis zum Olduvai-Event (Dodonov 1986); jedoch ist die dort dargestellte Abfolge (vgl. auch Pécsi \& Richter 1996, Abb. 101) von unserer $70 \mathrm{~m}$ mächtigen Sequenz wesentlich verschieden. Abb. 2 zeigt daher nur eine Gegenüberstellung der altpleistozänen Löß-BodenSequenzen von Luochuan und Chashmanigar. Dennoch ergibt der Vergleich, daß der altpleistozäne Abschnitt des Profils von Chashmanigar sehr viel mehr gut ausgebildete Paläoböden enthält als der entsprechende Abschnitt in Luochuan, insbesondere weil der „Wusheng“-Löß dort nur synsedimentär gebildete Paläoböden enthält (vgl. Kap. 3). Andere Lößprofile im chinesischen Lößplateau mögen im Altpleistozän reicher gegliedert sein, wie das in Baoji (Ding \& RutTer \& Liu, T. S. 1993), jedoch ist die Beschreibung und Darstellung aus paläopedologischer Sicht recht unbefriedigend. - Zum gegenwärtigen Kenntnisstand bietet die altpleistozäne Löß-Boden-Abfolge des Profils von Chashmanigar mehr paläoklimatische Informationen bezüglich kalt-trockener Phasen mit Lößakkumulation und feuchteren Warmzeiten, repräsentiert durch intensiv ausgebildeten Paläoböden als Lößprofile in China oder bisher bekannte $\partial 18 \mathrm{O}$-Tiefseekurven.

\subsection{Versuch einer Korrelation der Löß- Paläoboden-Sequenzen Zentralasiens mit $\partial$ 180-Tiefseekurven}

Das Sauerstoffisotopenverhältnis $18 \mathrm{O} / 16 \mathrm{O}(\partial 18 \mathrm{O})$, das sich z. B. in den Kalkschalen von Foraminiferen speichert, ist abhängig von der Größe der polaren Eisschilde: ihre Ausdehnung während der Eiszeiten führte zu höheren $\partial 18 \mathrm{O}$-Werten (EMiliani 1955, Shackleton \& Opdyke 1973). Somit spiegeln $\partial 18 \mathrm{O}$-Tiefseekurven insbesondere Temperaturkurven wider, wobei niedrige $\partial 18 \mathrm{O}-$ Werte einer Warmzeit, hohe $\partial 18 \mathrm{O}$-Werte einer Kaltzeit entsprechen.

Damit liegt es nahe, ว18O-Tiefseekurven LößPaläoboden-Sequenzen gegenüberzustellen, auch wenn letztere neben einer Temperaturkurve auch einen Wechsel im Feuchteregime - trockene Lößkaltzeiten im Wechsel mit feuchteren Warmzeiten während der Bodenbildung - widerspiegelt. Ein erster Vergleich der mittel- und jungpleistozänen Abfolge in Luochuan mit der astronomisch angepaßten SPECMAP-Kurve (IMBRIE et al. 1984) war im Ganzen bereits recht befriedigend (BRONGER \& HeinKele 1989a: Fig. 2). Ein früherer Versuch, die SPECMAP-Kurve auch mit der stärker gegliederten jung- und mittelpleistozänen Abfolge von Karamaydan zu vergleichen (BRONGER, et al. 1995: Figs. 3, 4, S. 79) ergab eine noch bessere Übereinstimmung. Die weiter verbesserte $\partial 18 \mathrm{O}$-Kurve von BAssinot et al. (1994) mit aus zyklischen Schwankungen der Erdbahnparameter abgeleiteten präzisen astronomischen Zeitangaben ermöglicht den Versuch einer noch genaueren Korrelation mit der mittel- und jungpleistozänen LößBoden-Abfolge des Referenzprofils von Karamaydan (Kap. 4.1). Wie für die pedostratigraphische Zuordnung zwischen den Abfolgen von Luochuan und Karamaydan (s. o.) dienen als Kontrollpunkte die Brunhes/Matuyama-Grenze sowie die Zuordnung des S 1-Pedokomplexes und des PK I mit dem $\partial 18$ O-Stadium 5 (vgl. auch Shackleton et al. 1995), wobei der jeweils basale Boden der beiden Pedokomplexe dem Substadium 5.5 zugeordnet wird (vgl. Abb. 1). Allerdings wird die Brunhes/Matuyama-Grenze bei Bassinot et al. (1994) in den unteren Teil des Stadiums 19 gestellt, während sie in den Profilen Tadjikistans und des chinesischen Lößplateaus im allgemeinen im Löß zwischen den PK IX und PK X bzw. zwischen dem S 7 und dem S 8 (PK) gefunden wurde (s. o.), der aller Wahrscheinkeit nach dem Stadium 20 entspricht (vgl. Abb. 1).

Dieser Versuch einer Korrelation erlaubt nun sogar eine Zuordnung von Böden innerhalb eines Pedokomplexes des Referenzprofils von Karamaydan zu Substadien der $\partial 18$ O-Kurve, für die genaue Angaben ihrer jeweiligen Modellalter (s. o.) vorliegen. Nur einige wenige der von BAssinot et al. (1994, Tab. 2) berechneten Altersangaben wurden hier ihrer $\partial 18 \mathrm{O}$-Kurve beigegeben ( $\mathrm{s}$. Abb. 1). Daraus läßt sich beispielsweise ablesen, daß die Bildung des unteren Bt-Horizontes des PK I entsprechend dem Substadium 5.5 bzw. dem Eem-Interglazial (i.e.S.) nur gut 10.000 Jahre gedauert hat, was z. B. mit den Ergebnissen von Jahresschichtenzählungen kombiniert mit Pollenanalysen an der eemzeitlichen Kieselgur von Bispingen (MülLER, 1974) übereinstimmt. Nach unseren paläopedologischen Untersuchungsergebnissen einer etwas höheren Tonbildungsrate verbunden mit deutlicher Tonverlagerung in dem basalen Bt-Horizont des PK I, verglichen mit dem holozänen Boden (s. Kap. 3) war das Klima des 
letzten Interglazials in diesem Teil Zentralasiens wahrscheinlich etwas feuchter als gegenwärtig. Dagegen erstreckte sich die Bildung der beiden Bt-Horizonte sowie des (A)B-Horizontes des PK II - unterbrochen durch zwei Abschnitte verstärkter äolischer Zufuhr - über etwa 50.000 Jahre, die der beiden Böden des PK III über ca. 15.000 bis 20.000 Jahre. - Im Stadium 11 bzw. 11.3, dem nach mehreren Autoren wärmsten Interglazial der letzten 1 Mill. Jahre (SHACKLETON 1987: 187) finden sich im PK IV in Karamaydan sogar ein Bt-, darüber ein B-Horizont. Die pedogene Tonbildungsrate von über $20 \%$, die deutlich höher als die des rezenten Bodens ist (s. Kap. 3; Details in Bronger \& Winter \& Sedov 1998, Fig. 4) dürfte wahrscheinlich ein Summeneffekt beider Bodenbildungsphasen, unterbrochen durch eine kurze Lößakkumulationsphase sein, die insgesamt mindestens 20.000 Jahre gedauert hat.

Anscheinend lassen sich z. B. auch die B- bzw. BtHorizonte des PK VI und des PK V gut mit den Substadien der Stufen 15 und 13 korrelieren. So ist z. B. der basale Bt-Horizont im PK VI einerseits nicht stärker verwittert als der holozäne Boden (s. Kap. 3, Abb. 3, 4). Andererseits ist dieser Boden(rest) mit mehr als 2,5 m Mächtigkeit für einen monogenetischen Boden viel zu mächtig. Unser Versuch einer Korrelation mit den Ergebnissen von BASSINOT et al. (1994) zeigt, daß sich dieser basale Bt-Horizont polygenetisch, wahrscheinlich unterbrochen durch eine äolische $\mathrm{Zu}$ fuhr (möglicherweise im Substadium 15.4) in einem Zeitraum von $\geq 20.000$ Jahren entwickelt hat. Auch unser Korrelationsversuch des kompliziert aufgebauten PK V mit den Substadien der Stufe 13 deutet darauf hin, daß sich dieser Pedokomplex mit drei Bt- bzw. B-Horizonten in einem Zeitraum von etwa 50.000 Jahren - unterbrochen jeweils durch äolische Zufuhr - gebildet hat (s. Abb. 1 und Abb. 4). - Die beiden Stadien 12 und 16, die nach SHACKLETON (1987: 187) extremen Glazialen entsprechen, sind im Profil von Karamaydan durch die beiden mächtigsten Stockwerke von typischen Lössen repräsentiert.

Noch problematisch ist dagegen eine Korrelation der altpleistozänen Löß-Paläoboden-Sequenz von Chashmanigar mit entsprechenden Abschnitten von $\partial 18 \mathrm{O}$-Tiefseekurven: dort wurde die Vorstellung der Dominanz von Kaltzeit-Warmzeit-Zyklen von 41.000 Jahren besonders für die Zeit $>1,2$ Mill. Jahren - im Unterschied zur Dominanz der 100.000-Jahres-Zyklen im Mittel- und Jungpleistozän - entwickelt (Ruddiman, RAYmo \& Me. Intyre, 1989, Berger u. Werer 1992). Entspre- chend werden für die Matuyama-Epoche (2,60,78 Mill. Jahre) die Stadien 104 bis 20 denen der Stadien 19 bis 1 (Holozän) gegenübergestellt (Tiedemann 1991; Tiedemann, Sarnthein \& ShackLETON 1994). Für die letzten 2,4 Mill. Jahre werden daher „zyklische Wechsel von insgesamt 55 Glazial- und Interglazial-Stadien" angenommen (Tiedemann 1995: 100) mit anscheinend aber geringeren Amplituden zwischen Kalt- und Warmzeiten in der Matuyama-Epoche. Diese Befunde und Schlußfolgerungen lassen sich mit unseren an der altpleistozänen Löß-Paläoboden-Abfolge in Chashmanigar (Abb. 2) (noch) nicht ohne weiteres in Einklang bringen: ähnlich wie im Mittelund Jungpleistozän sind auch im Altpleistozän die Paläoböden jedenfalls bis zum S XXVII - mit gleicher oder ähnlicher Verwitterungsintensität wie die mittel- und jungpleistozänen Böden (s. Kap. 3) - jeweils durch typische Lösse getrennt. Entsprechend unserer eingangs (Kap. 1) entwickelten Hypothese müssen andererseits die Klimaabläufe bzw. der Wechsel von Kalt- und Warmzeiten auch im Altpleistozän, dokumentiert in der Löß-Paläoboden-Abfolge bzw. in den entsprechenden Tiefseekurven, wie im Mittel- und Jungpleistozän zur Deckung gebracht werden können. Eine (nicht die einzige) Möglichkeit in Richtung auf eine Lösung könnte darin bestehen, daß wesentlich mehr altpleistozäne Paläoböden als oben vermutet (Kap. 3) polygenetische Pedokomplexe darstellen.

\subsection{Vergleich mit Löß-Paläoboden- Sequenzen Mitteleuropas}

Entsprechend unserer Hypothese (Kap. 1), daß sich jedenfalls im gemäßigten Klimagürtel der Nordhemisphäre Klimaschwankungen in der Zeitscheibe eines Glazial-Interglazial-Zyklus (ca. 105 Jahre) und aller Wahrscheinlichkeit nach auch der Länge eines Interglazials (ca. 104 Jahre) weitestgehend gleichzeitig stattfanden, soll schließlich ein pedostratigraphischer Vergleich mit Löß-Boden-Abfolgen Mitteleuropas versucht werden.

Die mittel- und jungpleistozänen Löß-Paläoboden-Sequenzen Ost- und Zentralasiens lassen sich mit denen etwa im jugoslawischen, kroatischen und ungarischen Teil des Karpatenbeckens, in Niederösterreich und Tschechien gut korrelieren, auch wenn einigen Pedokomplexen in Karamaydan „nur“ (scheinbar?) ungegliederte Paläoböden entsprechen. So lassen sich pedostratigraphisch die beiden Böden des PK I in Karamaydan (bzw. des S 1-Komplexes in Luo- 

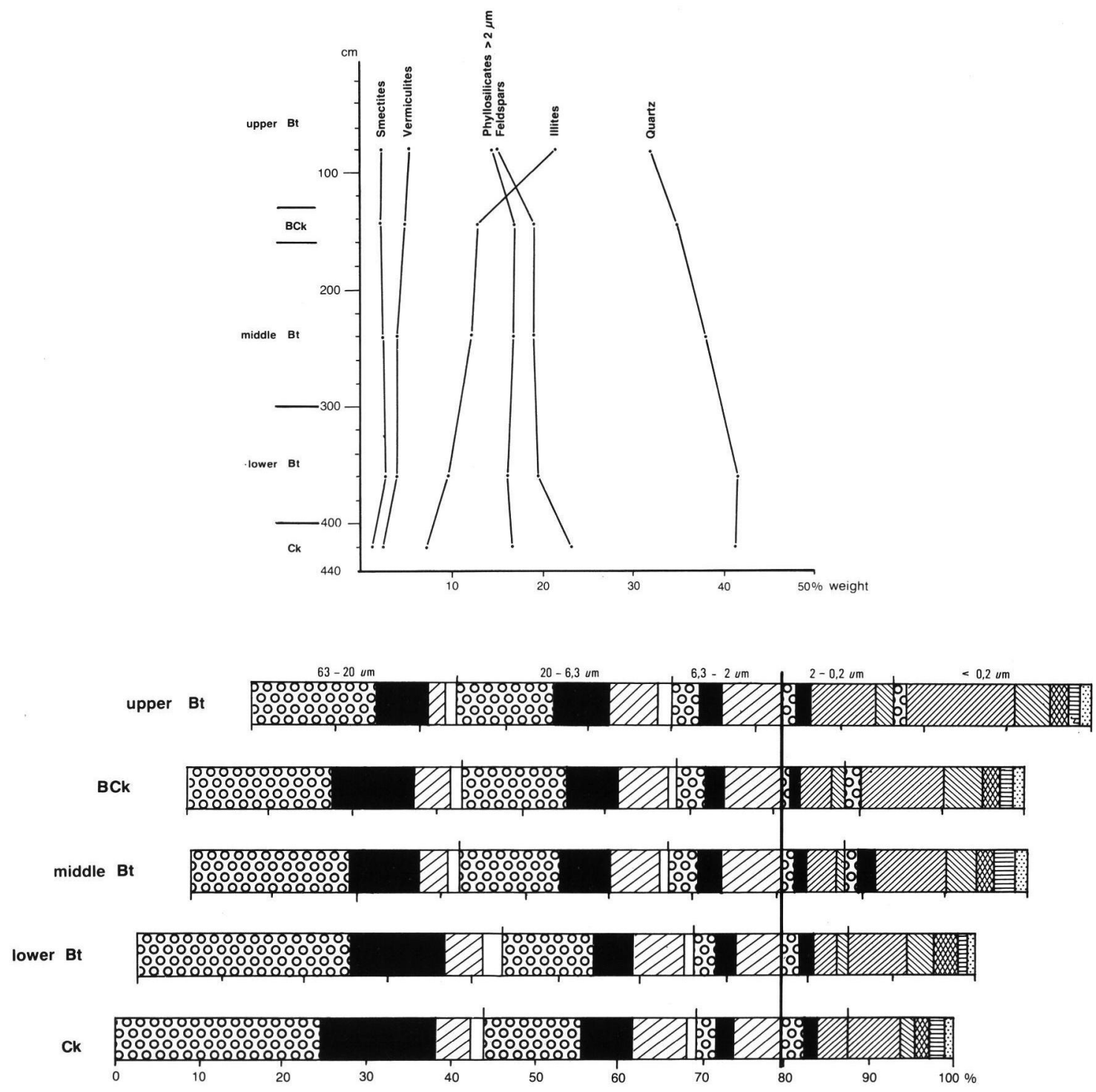

Abb. 5: Mineralogische und tonmineralogische Zusammensetzung des S5-Pedokomplexes in Luochuan/China. (vgl. Abb. 1 B; Lgende s. Abb. 3)

Fig. 5: Mineralogical and clay mineralogical composition of the S5-pedocomplex in Luochuan/China. (see fig. $1 \mathrm{~B}$; legend see fig. 3)

chuan) zwanglos mit dem F 2 und F 3 im Karpatenbecken (BRONGER \& HEINKELE 1989 b) korrelieren, besonders, seitdem der F 3 durch TL-Untersuchungen dem $\partial 18 \mathrm{O}$-Stadium 5.5 zugeordnet werden konnte (Singhvi et al. 1989, Singhvi in Bronger, 1995: 298-299). Aller Wahrscheinlichkeit nach entsprechen diese Böden dem PK II und PK III in Tschechien und Niederösterreich (zuletzt Kovanda \& SMOlikova \& HoraceK 1995), wobei bei der Korrelation mit dem PK II noch Diskussionsbedarf besteht (s. u.). Die Pedokom- plexe PK II bis PK IV in Karamaydan entsprechen aller Wahrscheinlichkeit nach den Böden F 4, F 5a und F 5b im Karpatenbecken (s. u.) bzw. den Pedokomplexen PK IV bis PK VI in Tschechien. Die Pedokomplexe PK V und PK VI parallelisieren wir mit dem F 6-Pedokomples (früher F 6, s. unten!) in Stari Slankamen bzw. mit dem PK VII und PK VIII in Tschechien. Der S VII und der (schwach entwickelte) S VIII sowie der PK IX in Karamaydan dürften pedostratigraphisch dem F 7 und F 8 im Karpatenbecken - 

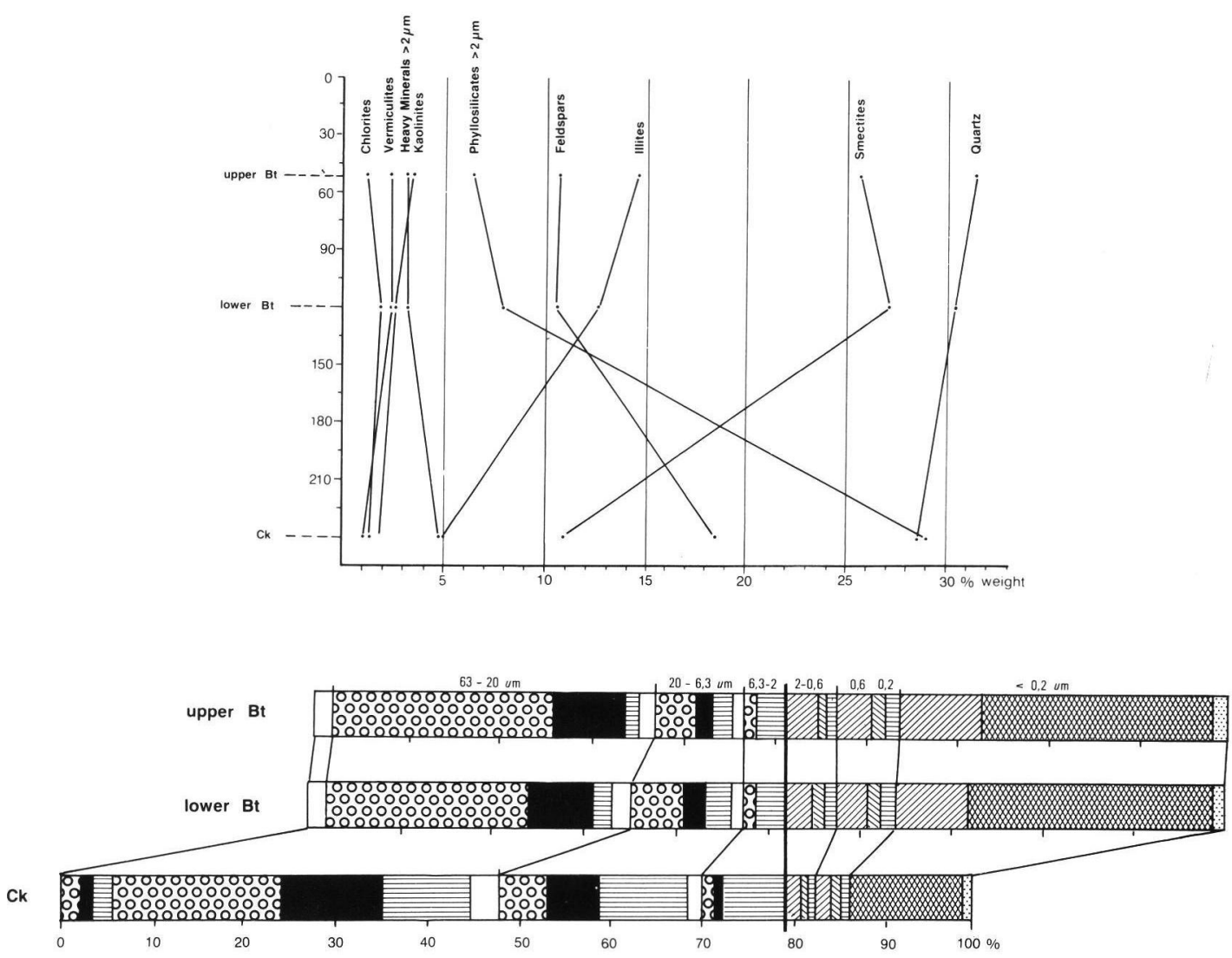

Primary Minerals $>2 \mu \mathrm{m}$

Clay Minerals $>2 \mu \mathrm{m}$
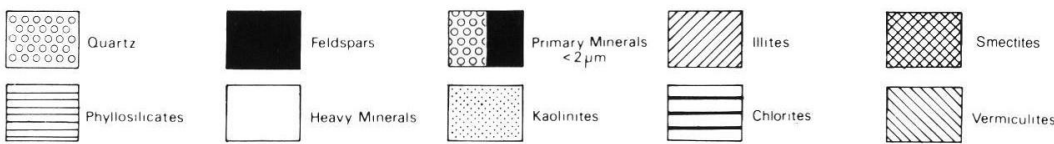

Abb. 6: Mineralogische und tonmineralogische Zusammensetzung des mittelpleistozänen rubefizierten F6-Bodenkomplexes im Lößprofil von Stari Slankamen/Jugoslawien. (Legende s. Abb. 3)

Fig. 6: Mineralogical and clay mineralogical composition of mid-Pleistocene strongly rubefied F6-pedocomplex of Stari Slankamen/Yugoslavia. (legend see fig. 3)

beide noch oberhalb der Brunhes/MatuyamaGrenze (Pécsi \& Pevzner, 1974, Marton 1979) bzw. den Pedokomplexen PK IX und PK X in Tschechien (s. Fig. 5 in Kovanda \& SMOlikova \& HORACEK 1995) entsprechen.

Es sei erwähnt, daß die Trennung des F 5-Bodens in einen $\mathrm{F} 5 \mathrm{a}$ und $\mathrm{F} 5 \mathrm{~b}$ im Karpatenbecken nur in den Lößprofilen von Neštin und Mende zu beobachten war (z. B. BRONGeR \& Heinkele 1989 b, Fig. 1). Oberhalb seines „BA“-Bodens - unser F 4 weicht die Löß-Boden-Stratigraphie ungarischer Aufschlüsse von Pécsi von der unsrigen ab. Allerdings finden sich im sehr bekannten Lößprofil von Paks in Pécsi \& Schwertzer (1995: 308-309) drei recht verschiedene Darstellungen. An ande- rer Stelle (PÉCsi \& Richter 1996: 107 und 150) unterscheiden sich die beiden Darstellungen des Profils von Paks wesentlich. Das ist für die chronostratigraphische Einordnung der Ergebnisse von TL-Untersuchungen, zusammengestellt in der zuletzt genannten Arbeit (a.a.O., S. 150), die jüngst um eine umfangreiche Studie erweitert wurde (Frechen \& HORVATH \& GABRIS 1997) sehr problematisch.

Unsere Schlußfolgerung aus den (ton)mineralogischen Untersuchungen an zwei holozänen Böden sowie zahlreichen Lössen und Paläoböden der Profile von Karamaydan und Chashmanigar (Bronger \& Winter \& Sedov 1998; Beispiele in Abb. 3, 4), daß die Klimate der Interglaziale im 
Jung-, Mittel- und Altpleistozän im Ganzen ähnlich wie das des Holozäns waren (Kap. 3) scheint im Widerspruch früherer Schlußfolgerungen für das südöstliche Mitteleuropa zu stehen (BRONGER 1976, Bronger \& Heinkele 1989b): aus zwar gleicher Art, aber wesentlich größerem Ausmaß der Mineralverwitterung und Tonmineralbildung eines mittelpleistozänen und einiger altpleistozäner rubefizierter erdiger Braunlehme im Vergleich zu holozänen klimaphytomorphen Lößböden wurde auf ein wärmeres, wahrscheinlich subtropisches Klima in diesem Raum geschlossen. Als Beispiel zeigt der mittelpleistozäne F6-Boden (s. o.) in Stari Slankamen eine wesentlich stärkere pedochemische Verwitterung und Tonmineralbildung als die holozänen Böden - Tschernoseme bis Phaeozeme - der Umgebung: mehr als $40 \%$ der Feldspate und fast $80 \%$ der Glimmer in den Schluff-Fraktionen sind abgebaut worden (s. Abb. 6); in Dünnschliffen sind nur noch Muskovite, keine Biotite mehr zu finden. Die Bildung von Illiten und besonders Smectiten ist erheblich höher als in holozänen Böden; die pedogene Kaolinitbildung blieb dagegen gering. Später (BRONGER \& Heinkele 1989b) wurde dieser F 6-Boden mit dem $\mathrm{S}$ 5-Pedokomplex in Luochuan pedostratigraphisch korreliert, dieser hier mit dem PK VI und dem PK V in Karamaydan (Kap. 4.1 und Abb. 1B). Selbst im mächtigen basalen Bt-Horizont des PK VI ist die Mineralverwitterung und Tonmineralbildung nicht oder unwesentlich höher als in den holozänen Böden; das gleiche gilt für den basalen Bt-Horizont des PK V (vgl. Abb. 3, 4).

Auch Art und Ausmaß der Mineralverwitterung und Tonmineralbildung im S 5-Pedokomplex in Luochuan, selbst im oberen, stärker rubefizierten Bt-Horizont (Abb. 5) bleibt im Rahmen der der holozänen Böden dieses Raumes (BRONGER \& Heinkele 1989a, Heinkele, 1990). Zusammenfassend läßt sich folgern, daß das viel größere Ausmaß der pedochemischen Verwitterung und Tonmineralbildung im F 6 von Stari Slankamen nicht die Folge eines wärmeren Klimas, sondern in erster Linie des bodenbildenden Faktors Zeit ist: dieser Boden entspricht aller Wahrscheinlichkeit nach chronostratigraphisch - via dem S 5-Pedokomplex in Luochuan - mindestens fünf B- bzw. Bt-Horizonten, zusammengefaßt zu den Pedokomplexen VI und $\mathrm{V}$ in Karamaydan. Unter $\mathrm{Zu}-$ hilfenahme der $\partial 18 \mathrm{O}$-Kurve von BAssinot et al. (1994; vgl. Kap. 4.2) läßt sich folgern, daß die Pedokomplexe VI und V in einem Zeitraum von ca. 140.000 Jahren gebildet wurden, wobei die Zeiten der Bodenbildung mehrfach durch Zeiten der
Lößakkumulation unterbrochen waren. Insgesamt standen für die Bildung des F 6 , besser bezeichnet als F 6-Bodenkomplex ein Mehrfaches der Zeit des Holozäns zur Verfügung, was die viel höhere Rate der pedochemischen Mineralverwitterung und Tonmineralbildung erklärt.

Aus dem Vergleich mit der detaillierten altpleistozänen Löß-Boden-Abfolge von Chashmanigar, die wahrscheinlich bis etwa zum Olduvai-Event reicht, aber sicher nicht vollständig ist (Kap. 4.1, 4.2), mit Böden, deren Verwitterungsintensität im Rahmen der des Holozäns liegt (Kap. 3) folgt einmal, daß die altpleistozänen Löß-Boden-Sequenzen im Karpatenbecken äußerst lückenhaft sind. Das hat seine Ursache in der sehr labilen morphotektonischen Struktur dieses Raumes (zus.fass. BRONGER 1976). Aller Wahrscheinlichkeit nach müssen die rubefizierten erdigen Braunlehmreste F 9, F 10, und F 11 in Stari Slankamen als Reste ehemaliger Pedokomplexe angesehen werden. - In Niederösterreich sind dagegen die altpleistozänen Löß-Boden-Abfolgen wesentlich detaillierter erhalten, dazu paläomagnetisch „datiert", vor allem in Krems-Schießstätte und in Stranzendorf (KOvanda \& SMOLIKOVA \& HORACEK 1995); im letzteren Profil sind sogar in der GaussEpoche drei „Rotlehme“, getrennt durch zwei Lösse erhalten (a. a. O., Abb. 3). Sowohl in der prä-Olduvai-zeitlichen Abfolge in Stranzendorf wie auch in der Abfolge in Krems, die vom Olduvai-Event bis in den untersten Abschnitt der Brunhes-Epoche reicht, werden die Paläoböden stets als „Braunlehme“, z. T. später rubefiziert, gelegentlich als „Rotlehme“ angesprochen. Hierfür wurde ein Teil der Böden mikromorphologisch charakterisiert; weitere analytische, z. B. granulometrisch oder (ton)mineralogische Untersuchungen vermißt man sehr. Auch weil die Löß-BodenAbfolge des oberen Altpleistozäns in KremsSchießstätte (s. o.) deutlich weniger gegliedert ist als die in Chashmanigar bleibt die Frage, ob die vor allem im Vergleich mit den holozänen Böden dieses Raumes - anscheinend viel stärker ausgeprägten Paläoböden in Krems nicht jeweils das Ergebnis länger andauernder Bodenbildungsphasen, möglicherweise unterbrochen durch Lößakkumulation gewesen sind, vielleicht ähnlich der Bildungsgeschichte des F6-Komplexes (s. o.). Auch der paläoklimatische Aussagewert der Ferretto-Böden könnte eine andere Deutung zulassen: diese weitverbreiteten rotbraunen Böden (5 YR in der MunSELL-Skala) aus Sanden bis Schottern werden in Südmähren dem „siallitischallitischen" Verwitterungstyp zugeordnet (SMOLI- 
Kova \& Zeman 1982). Sie bildeten sich zuletzt im "Comer-Interglazial“, das in seinem Klimaoptimum um $4^{\circ}$ wärmer war als das Holozän“; diese Böden „haben im Mittel- und Jungpleistozän keine Analogie mehr" (a. a. O., S. 81). Auch hier könnte der bodenbildende Faktor Zeit, nicht ein wärmeres Klima der wesentliche Faktor gewesen sein, zumal das „Cromer-Interglazial“ d. h. der Cromer-zeitliche Interglazialkomplex ( „Cromerium", zus.fass. URBan 1997) von der d18O-Stufe 21 bis Stufe 13, vielleicht sogar bis zur Stufe 11 ( $\mathrm{ZAG}^{-}$ WIJN 1992, Fig. 9), d. h. insgesamt mindestens 350.000 Jahre gedauert hat.

Abschließend soll erwähnt werden, daß für kleinere Zeitscheiben eine Korrelation von LößPaläoboden-Sequenzen mit der $\partial 18 \mathrm{O}-T i e f s e e-$ kurve bzw. ein kontinentaler Vergleich dieser Sequenzen noch auf Schwierigkeiten stößt. Das gilt z. B. für das Frühwürm, das in den SauerstoffIsotopenkurven durch die Substadien 5.3 und 5.1 (bzw. 5c und 5a) gekennzeichnet ist. In verschiedenen Teillandschaften Mitteleuropas einschließlich der Niederlande und Belgiens sind in diesem Zeitabschnitt in zahlreichen Aufschlüssen drei „Humuszonen“ entwickelt (zus.fass. SEmmel 1989). In Mainz-Weisenau waren die beiden basalen „Mosbacher Humuszonen" sogar entkalkt, mit Ausbildung eines Ck-Horizontes (SEmmel 1995, Bibus et al. 1996). Nach pollenanalytischen und malakkologischen Untersuchungen konnten die drei Humuszonen nach den zuletzt genannten Autoren jeweils als Interstadiale mit wahrscheinlich einem kühlgemäßigt kontinentalen Klima charakterisiert werden, in denen sich wieder Wald ausgbreitet hatte. Nach TL-Untersuchungen (ZÖlLER 1995: 154, FreChEN \& Preusser 1996: 58 ff, jeweils mittlere Alter) fiel die Bildung der Böden in den Zeitraum zwischen etwa 110.000 bis etwa 65.000 Jahre v. h. Wahrscheinlich entsprechen diese drei Böden pedostratigraphisch dem oberen Ah-Horizont des PK III (der nicht zum liegenden interglazialen Bt-Horizont gehört) sowie den beiden ebenfalls entkalkten Ah-Horizonten des PK II (s. o.) in Dolni Vestonice /Südmähren (vgl. Bronger 1976: 146-149); ihr TL-Alter konnte kürzlich zwischen ca. 102.000 und ca. 55.000 Jahre eingegrenzt werden (SinghVI in Bronger 1995: 228). Dagegen ist in den Lößaufschlüssen des Karpatenbeckens in dieser stratigraphischen $\mathrm{Po}_{\mathrm{O}}$ sition nur ein Boden, der F 2 (s. o.) vorhanden: seine intensivere Ausprägung, die oft an die des letztinterglazialen F 3-Bodens bzw. an die des holozänen klimaphytomorphen Bodens dieses Raumes heranreicht, ist wahrscheinlich auch eine
Folge einer längeren Bildungszeit. Wie oben kurz dargelegt, ist oberhalb des letztinterglazialen Bodens in Luochuan und weiteren Aufschlüssen des chinesischen Lößplateaus, sowie z. B. in Karamaydan (vgl. Abb. 1 A) auch jeweils nur ein Boden vorhanden. Dagegen sind im zentralasiatischen, ebenfalls winterfeuchten Kashmir Valley oberhalb des letztinterglazialen Bt-Horizontes wie im westlichen Mitteleuropa - drei gut ausgebildete Humuszonen bzw. Ah-Horizonte, z. T. mit Tonverlagerung, dann Aht-Horizonte, aber nur in 30-50 cm Mächtigkeit erhalten (BRONGER \& PANT \& SinghVI 1987). Ihre Bildungszeit fällt nach TLUntersuchungen (ebenfalls) in den Zeitraum zwischen ca. 80.000 und 50.000 Jahre v. h. (Singhvi et al. 1987).

Für diese unterschiedlichen Löß-Boden-Abfolgen im Frühwürm in benachbarten Räumen einerseits, großer Ähnlichkeit in sehr weit voneinander entfernt liegenden Räumen recht verschiedener Klimate andererseits, die sich in den Tiefseekurven (noch) nicht ausreichend abbilden, besteht noch einiger Erklärungsbedarf. Für größere Zeitscheiben von ca. $10^{5}$ Jahren zeigt unser Versuch einer engen Korrelation von Löß-Paläoboden-Sequenzen mit der $\partial 18 \mathrm{O}$-Tiefseekurve, daß wir der tatsächlichen Abfolge von Kalt- und Warmzeiten für das Mittel- und Jungpleistozän wahrscheinlich recht nahe gekommen sind. Sie müssen im gemäßigten Klimabereich der Nordhemisphäre weitestgehend gleichzeitig stattgefunden haben. Von der entsprechenden näheren Kenntnis einer Klimageschichte der Matuyama-Epoche bzw. des Altpleistozäns sind wir noch ein gutes Stück entfernt.

\section{Danksagung}

Für die finanzielle Unterstützung der Geländeuntersuchungen, der mikromorphologischen sowie der Laborarbeiten des Projektes „Quartär Tadjikistan" (Br 303/26-1 bis 3) bin ich der DFG sehr dankbar. Entsprechende frühere Untersuchungen im Projekt „Quartäre Klimaentwicklung - China“ (Br 303/20-1 und 2) wurden ebenfalls dankenswerterweise von der DFG finanziell unterstützt.

\section{Schriftenverzeichnis}

BARshaD, I. (1967): Chemistry of Soil Development. In: Bear, F.E. (Ed.): Chemistry of the Soil, New York Reinhold: 1-70.

Bassinot, F.V., Labeyrie, L.D., Vincent, E., Quidelleur, X., Shackleton, N.J. \& Lancelot, Y. (1994): The astronomical theory of climate and the age of Brunhes-Matuyama magnetic reversal. Earth and Planetary Science Letters, 126: 91-108. 
Bibus, E., Bludau, W., Bross, C., Rählen, W. (1996): Der Altwürm- und Rißabschnitt im Profil Mainz - Weisenau. Frankfurter Geowiss. Arb. D 20: 21-52.

Brewer, R. (1964): Fabric and Mineral Analysis of Soils. New York: John Wiley \& Sons. 470pp.

Bronger, A. (1976): Zur quartären Klima- und Landschaftsgeschichte des Karpatenbeckens auf paläopedologischer und bodengeographischer Grundlage. Kieler Geographische Schriften, 45: 1-268. 13 Abb., 24 Farbbilder.

- (1991): Argillic horizons in modern loess soils in an ustic soil moisture regime? Comparative studies in forest- steppe and steppe areas from Eastern Europe and the USA. Advances in Soil Science, 15: 41-90. (incl. 2 soil maps).

- (1995): Introduction - Some chronostratigraphical problems in eastern Central Europe. In: Schirmer, W. (Ed.): Quaternary field trips in Central Europe, Vol. I: 298-305, München.

— \& Heinkele, TH. (1989 a): Micromorphology and genesis of paleosols in the Luochuan loess section, China: Pedostratigraphic and environmental implications. Geoderma, 45: 123-143.

— \& Heinkele, Th. (1989 b): Paleosol sequences as witnesses of Pleistocene climatic history. In: A. ronger \& J.Catt (Eds.): Paleopedology - Nature and Application of Paleosols. Catena Supplement, 16: 163-186.

- \& Heinkele, Th. (1990): Mineralogical and Clay Mineralogical Aspekts of Loess Research. Quaternary International, 7/8: 37-51.

- Kalk, E. \& Schröder, D. (1976): Über Glimmer- und Feldspatverwitterung sowie Entstehung und Umwandlung von Tonmineralen in rezenten und fossilen Lößböden. Geoderma, 16: 21-54.

- Pant, R. K. \& Singhvi, A. K. (1987): Pleistocene Climatic Changes and Landscape Evolution in the Kashmir Basin, India: Paleopedologic and Chronostratigraphic Studies. - Quaternary Research, 27: 167-181.

- Winter, R., Derevjanko, O. \& Aldag, S. (1995): LoessPalaeosol-Sequences in Tadjikistan as a Palaeoclimatic Record of the Quaternary in Central Asia. Quaternary Proceedings, 4: 69-81.

- Winter, R. \& Heinkele, Th. (1998): Pleistocene Climatic History of East and Central Asia based on paleopedological indicators in Loess Paleosol Sequences. Catena, 34: 1-17.

- Winter, R. \& Sedov, S. (1998): Weathering and clay mineral formation in two holocene soils and in buried palaeosols in Tadjikistan: Towards a Quaternary Paleoclimatic Record in Central Asia. Catena, 34: 19-34.

Ding, Z., RutTer, N. \& Liu, T.S. (1993): Pedostratigraphy of Chinese Loess Deposits and Climatic Cycles in the last 2,5 Myr. Catena, 20: 73-91.

Dodonov, A. E. (1979): Stratigraphy of the Upper Pliocene- Quaternary Deposits of Tajikistan (Soviet Central Asia). Acta Geologica Academiae Scientarum Hungaricae, 22 (1-4): 63-73.

- (1984): Stratigraphy and Correlation of Upper Pliocene-Quaternary deposits of Central Asia. In: Pecsi, M. (Ed.): Lithology and Stratigraphy of Loess and Paleosols, Budapest: 201-211.
- (1986): Stratigraphy and Paleogeography of Loess in Middle Asia. Annales Universitatis Mariae CurieSklodowska Lublin-Polonia, Vol. XII,1: 1-14.

- (1991): Loess of Central Asia. Geojournal, 24(2): 185-194.

Emiliani, C. (1955): Pleistocene temperatures. Journal of Geology, 63: 538-578.

Forster, TH. \& Heller, F. (1994): Loess deposits from the Tajik depression (Central Asia): Magnetic properties and paleoclimate. Earth and Planetary Science Letters, 128: 501- 512.

Frechen, M., Horvarth, E. \& Gabris, G. (1997): Geochronology of Middle and Upper Pleistocene Loess Sections in Hungary. Quaternary Research, 48: 291-312.

- Dodonov, A. E. (1998): Loess chronology of the Middle and Upper Pleistocene in Tadjikistan. Geol. Rundsch. 87: 2-20.

- Preusser, F. (1996): Kombinierte Lumineszenz - Datierungen am Beispiel des Lößprofils Mainz - Weisenau. Frankfurter Geowiss. Arb. D 20: 53-66.

Heinkele, T. (1990): Bodengeographische und paläopedologische Untersuchungen im zentralen Lößplateau von China - ein Beitrag zur quartären Klimaund Landschaftsgeschichte. Schriftenreihe Inst. f. Pflanzenernährung und Bodenkunde $d$. Universität Kiel, 9: 1-120.

Heller, F. \& Liu, T. S. (1982): Magnetostratigraphical dating of loess deposits in China. Nature, 300: 431-432.

- \& Liu, T. S. (1984): Magnetism of Chinese loess deposits. Geophys. J. R. Astron. Soc., 77: 125-141.

imbrie, J., Hays, J. D., Martinson, D. G., Mcintyre, A., Mix, A. C., Morley, J. J., Pisias, N. G., Prell, W. L. \& Shackleton, N. J. (1984): The Orbital Theory of Pleistocene Climate: Support from a Revised Chronologie of the Marine 2180 Record. In: Berger, A., Imbrie, J., Hays, J., Kukla, G. \& Saltzman, B. (Eds.): Milankovitch and Climate: 269-305.

ISSS-ISRIC-FAO (1994): World Reference Base for Soil Resources (Draft). (Compiled and edited by O.C. Spaargaren). Wageningen, Rome. 161pp.

Kovanda, J., Smolikova, L., HoraceK, I. (1995): New Data on four classic loess sequences in Lower Austria. Anthropozoikum, 22: 63-85. Praha.

Lazarenko, A. A. (1984): The Loess of Central Asia. In: Velichko: Late Quaternary Environments of the Soviet Union, Minneapolis: University of Minnesota Press: $125-131$.

Liu, T. S. et al. (1985): Loess and the Environment. Beijing. 251p.

Liu, X. M., Liu, T. S., Shaw, J., Heller, F., Xu, T. \& Yuan, B. (1991): Paleomagnetic and paleoclimatic studies of Chinese loess. In: Liu, T.S. (Ed.): Loess, Environment and Global Change, Beijing: Science Press: 61-81.

Lomov, S. P. (1985): Soils of the major typical landscapes of Hissar nature economic field. Dushanbe (Donish Publishers), Tadjik. Academy of Sciences.

Maisch, M. (1995): Alpine Traverse. In: Schirmer, W. (Ed.): Quaternary field trips in Central Europe, Vol. II: $655-705$, München. 
Marton, P. (1979): Paleomagnetism of the Paks brickyard exposures. Acta Geologica Acad. Sci. Hung. 22 (1-4): 445-449.

Müller, H. (1974): Pollenanalytische Untersuchungen und Jahresschichtenzählungen an der eemzeitlichen Kieselgur von Bispingen / Luhe. Geol. Jahrb., A 21: 149-169.

PÉcsi, M. \& SchweItzer, F. (1995): The development of the Hungarian Lowland and Upland section of the Danube valley. In: Schirmer, W. (Ed.): Quaternary field trips in Central Europe Vol. I: 305- 317, München.

— \& Pevzner, M. A. (1974): Paleomagnetic measurements in the loess-sequences at Paks and Dunaföldvar, Hungary. Földr. Közlem.: 215-219.

— \& Richter, G. 1996. Löss. Herkunft, Gliederung, Landschaften. Z. Geomorphologie, N.F., Suppl.Bd 98: 1-391. Berlin, Stuttgart.

Penkov, A.V. \& Gamov, L. N. (1977): Paleomagnetic Datums in Pliocene-Quaternary Strata of Southern Tajikistan. Abstracts of the IGCP International Symposium on the Neogene-Quaternary Boundary: 46-47. Moscow.

- Gamov, L. N. (1980): Paleomagnetic datums in the Pliocene to Quaternary strata of southern Tajikistan. The Neogene-Quaternary boundary (IGCP Project Nr.41), Nauka, Moscow, 189-194.

Ruddiman, W.F., Raymo, M., McIntyre, A. (1989): Matuyama 41,000- year cycles: North Atlantic Ocean and northern hemisphere ice sheets. Earth Planet. Sci. Letters, 80: 117-129.

SCHRÖDER, D. (1992): Bodenkunde in Stichworten. Berlin, Stuttgart: Hirtverlag.

Semmel, A. (1989): Paleopedology and Geomorphology: Examples from the western part of Central Europe. In: A. Bronger \& J. CATt (Eds.): Paleopedology Nature and Application of Paleosols. Catena Supplement, 16: 143-162.

- (1995): Stop 14: Quarry of the Portlandzementwerke Heidelberg at Mainz-Weisenau. In: Schirmer, W. (Ed.): Quaternary field trips in Central Europe, Vol. I: 452-454, München.

Shackleton, N. J. (1987): Oxygen isotopes, ice volume and sea level. Quat.Sci.Reviews, Vol. 6: 183-190.

- \& Opdyke, N. D. (1973): Oxygene Isotope and Paleomagnetic Stratigraphy of Equatorial Pacific Core V28-238: Oxygen Isotope Temperature and Ice Volumes on a $10^{5}$ and $10^{6}$ Year Scale. Quaternary Research, 3: 39-55.

- An, Z., Dodonov, A. E., Gavin, J., Kukla, G. J., RANOv, V. A. \& ZHOU, L. P. (1995): Accumulation Rate of Loess in Tadjikistan and China: Relationship with Global Ice Volume Cycles. - Quat. Proc., 4: 1-6.

Singhvi, A. K., Bronger, A., Sauer, W. \& Pant, R. K. (1989): Thermoluminescence dating of loess-paleosol sequences in the Carpathian Basin (East-Central Europe): A suggestion for a revised chronology. Chemical Geology (Isotope Geoscience Section), 73: 307-317
Smolikova, L. (1967): Polygenese der fossilen Lößböden der Tschechoslowakei im Lichte mikromorphologischer Untersuchungen. Geoderma, 1: 315-324.

- (1971): Gesetzmäßigkeiten der Bodenentwicklung im Quartär. Eiszeitalter u. Gegenwart, 22: 156-177.

- (1990): Problematika paleopedologie. Regionalni paleopedologie. In: Nemecex, J., Smolikova, L. \& KuTILEK, M. (EDS.): Pedologie a paleopedologie, Praha: 381-479.

- Zeman, A. (1982): Bedeutung der Ferretto-Böden für die Quartärgeographie. Anthropozoikum, 14: 57-93. Praha

SOIL SURVEY STAFF (1975): Soil Taxonomy. A Basic System of Soil Classification for Making and Interpreting Soil Surveys (Agriculture Handbook No. 436). Washington, D.C.:U.S. Govt. Printing Office. U.S.D.A. Soil Conservation Service.

- (1996): Keys to Soil Taxonomy (7th Edition). USDA. Soil Conservation Service - U.S. Government Printing Office.

STANinkovitch, K. (1968): Geobotanic zonation of Tadjikistan. Atlas of Tajik SSR. Academy of Sciences of Tajik SSR.

Tiedemann, R. (1991): Acht Millionen Jahre Klimageschichte von Nordwest-Afrika und Paläo-Ozeanographie des angrenzenden Atlantiks: Hochauflösende Zeitreihen von ODP-Sites 658-661. Berichte-Reports d. Geol.-Paläont. Inst. d. Univ. Kiel, 46: 1-190. Kiel.

- (1995): Meeressedimente - Zeugen der Ozeanund Klimageschichte. Geograph. Rundschau, 47: 97-104.

- Sarnthein, M., Shackleton, N. J. (1994): Astronomic time scale for the Pliocene Atlantic $218 \mathrm{O}$ and dust flux records of Ocean Drilling Program site 659. Paleoceanography, Vol. 9, No. 4: 619-638.

URBAN, B. (1997): Grundzüge der eiszeitlichen Klimaund Vegetationsgeschichte in Mitteleuropa. In: Wagner, G.A. \& Beinhauer, K.W. (Eds.): Homo heidelbergensis von Mauer. Das Auftreten des Menschen in Europa, 241-263, Heidelberg.

ZAGWIJN, W. H. (1992): The Beginning of the Ice Age in Europe and its major subdivisions. Quatern. Sci. Rev., Vol. 11: 583-591.

Zapriagaeva, V.I. (1964): Wildgrowing fruits of Tajikistan. 695p. Moscow, Leningrad (russ.).

- (1968): Map of wildgrowing fruits, 1:1,5 Mill. Atlas of Tajik SSR, Academy of Sciences of Tajik SSR, Moscow, Leningrad, (russ.).

ZÖLlER, L. (1995): Würm- und Rißlöß. - Stratigraphie und Thermolumineszenz-Datierung in Süddeutschland und angrenzenden Gebieten. Habil.schrift.Fak. Geowiss. Univ. Heidelberg (unveröff.).

Manuskript eingegangen am 14. Juli 1998 